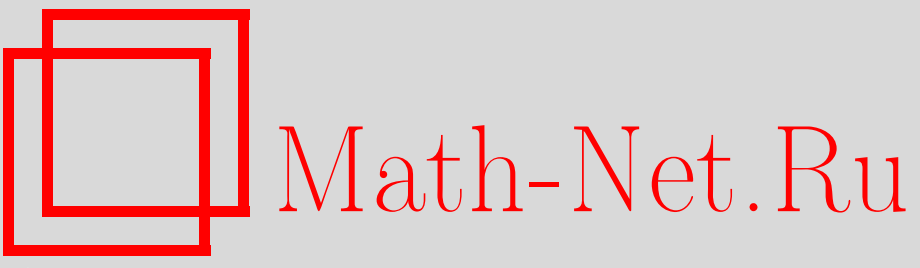

A. A. Mogul'skiǐ, E. I. Prokopenko, Local theorems for arithmetic multidimensional compound renewal processes under Cramér's condition, Mat. Tr., 2019, Volume 22, Number 2, 106-133

DOI: https://doi.org/10.33048/mattrudy.2019.22.207

Use of the all-Russian mathematical portal Math-Net.Ru implies that you have read and agreed to these terms of use

http: //www.mathnet.ru/eng/agreement

Download details:

IP : 35.173 .219 .12

April 26, 2023, 18:12:01 


\title{
ЛОКАЛЬНЫЕ ТЕОРЕМЫ ДЛЯ АРИФМЕТИЧЕСКИХ МНОГОМЕРНЫХ ОБОБЩЕННЫХ ПРОЦЕССОВ ВОССТАНОВЛЕНИЯ ПРИ ВЫПОЛНЕНИИ УСЛОВИЯ КРАМЕРА
}

\author{
А. А. Могульский, Е. И. Прокопенко
}

В настоящей работе продолжается изучение обобщенных процессов восстановления (о.п.в.) при выполнении моментного условия Крамера, начатое в [1-9; 11-15]. Изучаются два типа арифметических многомерных о.п.в. $\mathbf{Z}(n)$ и $\mathbf{Y}(n)$, для которых случайный вектор $\xi=(\tau, \boldsymbol{\zeta})$, «управляющий» этими процессами $(\tau>0$ определяет расстояние между скачками, $\boldsymbol{\zeta}$ определяет величину скачков о.п.в.), имеет арифметическое распределение и удовлетворяет моментному условию Крамера. Для этих процессов найдены точные асимптотики в локальных предельных теоремах для вероятностей

$$
\mathbb{P}(\mathbf{Z}(n)=\boldsymbol{x}), \quad \mathbb{P}(\mathbf{Y}(n)=\boldsymbol{x})
$$

во всей крамеровской зоне уклонений $\boldsymbol{x} \in \mathbb{Z}^{d}$ (в $[8 ; 9 ; 12-14]$ аналогичная задача решена для нерешетчатых о.п.в., когда вектор $\boldsymbol{\xi}=$ $(\tau, \boldsymbol{\zeta})$ имеет нерешетчатое распределение).

Ключевые слова и фразы: обобщенный процесс восстановления; моментное условие Крамера; арифметическое распределение; функция восстановления; функция уклонений; большие уклонения; умеренно большие уклонения; локальная предельная теорема.

\section{§1. Введение. Постановка задачи}

Условимся элементы $d$-мерного евклидова пространства $\mathbb{R}^{d}$ обозначать полужирными символами, например, $\boldsymbol{x}=\left(x_{(1)}, \ldots, x_{(d)}\right), \mathbf{0}=(0, \ldots, 0)$, $\boldsymbol{\mu}=\left(\mu_{(1)}, \ldots, \mu_{(d)}\right)$. Скалярное произведение для элементов $\boldsymbol{x}, \boldsymbol{y} \in \mathbb{R}^{d}$ будем обозначать как

$$
\boldsymbol{x y}:=x_{(1)} y_{(1)}+\cdots+x_{(d)} y_{(d)} .
$$

Работа выполнена при финансовой поддержке Российского научного фонда (проект № 18-11-00129).

(C) А. А. Могульский, Е. И. Прокопенко; 2019 
Норму в $\mathbb{R}^{d}$ обозначим через $|\boldsymbol{x}|:=\sqrt{\boldsymbol{x} \boldsymbol{x}}$. Случайные векторы со значениями в $\mathbb{R}^{d}$ тоже будем обозначать полужирными буквами, например, $\boldsymbol{\zeta}=\left(\zeta_{(1)}, \ldots, \zeta_{(d)}\right)$. Через

$$
\boldsymbol{\xi}=(\tau, \boldsymbol{\zeta})=\left(\tau, \zeta_{(1)}, \ldots, \zeta_{(d)}\right)
$$

будем обозначать случайный вектор в пространстве $\mathbb{R}^{d+1}$.

Рассмотрим последовательность

$$
\left\{\boldsymbol{\xi}_{k}=\left(\tau_{k}, \boldsymbol{\zeta}_{k}\right)\right\}_{k=0}^{\infty}
$$

независимых случайных векторов в пространстве $\mathbb{R}^{d+1}$ таких, что $\tau_{0}=0$, $\boldsymbol{\zeta}_{0}=\mathbf{0}, \tau_{1} \geq 0, \tau_{k}>0$ при $k \geq 2$. Пусть векторы $\boldsymbol{\xi}_{k}=\left(\tau_{k}, \boldsymbol{\zeta}_{k}\right)$ при $k \geq 2$ имеют общее распределение с вектором $\boldsymbol{\xi}=(\tau, \boldsymbol{\zeta})$. Положим

$$
T_{n}:=\sum_{k=0}^{n} \tau_{k}, \quad \mathbf{Z}_{n}:=\sum_{k=0}^{n} \boldsymbol{\zeta}_{k}, \quad \mathbf{S}_{n}=\left(T_{n}, \mathbf{Z}_{n}\right):=\sum_{k=0}^{n} \boldsymbol{\xi}_{k} \text { при } n \geq 0,
$$

так что $T_{0}=0, \mathbf{Z}_{0}=\mathbf{0}, \mathbf{S}_{0}=(0, \mathbf{0})$. Пусть $\nu(0)=\eta(0):=0$. При $t>0$ положим

$$
\nu(t):=\max \left\{k \geq 0: T_{k}<t\right\}, \quad \eta(t):=\min \left\{k \geq 0: T_{k} \geq t\right\},
$$

так что $\eta(t)=\nu(t)+1$ при $t>0$.

Первыц и второй обобщенные процессы восстановления (о.п.в.) $\mathbf{Z}(t)$, $\mathbf{Y}(t)$ определяются равенствами (см., например, [1; 10])

$$
\mathbf{Z}(t):=\mathbf{Z}_{\nu(t)}, \quad \mathbf{Y}(t):=\mathbf{Z}_{\eta(t)}, \quad t \geq 0,
$$

соответственно. Стандартная общепринятая модель о.п.в. предполагает, что время $\tau_{1}$ появления первого скачка и величина $\boldsymbol{\zeta}_{1}$ этого скачка имеют совместное распределение, отличное, вообе говоря, от совместного распределения $(\tau, \boldsymbol{\zeta})$ (см., например, [16]). Это реализуется, например, для о.п.в. со стационарными приращениями.

Если $\left(\tau_{1}, \boldsymbol{\zeta}_{1}\right) \stackrel{d}{=}(\tau, \boldsymbol{\zeta})$ (или, что то же самое, $\left.\left(\tau_{1}, \boldsymbol{\zeta}_{1}\right) \stackrel{d}{=}(0, \mathbf{0})\right)$, то процессы $\mathbf{Z}(t)$ и $\mathbf{Y}(t)$ будем называть однородными о.n.в.; в противном случае неоднородными.

Итак, распределения двух случайных векторов $\boldsymbol{\xi}_{1}=\left(\tau_{1}, \boldsymbol{\zeta}_{1}\right), \boldsymbol{\xi}=(\tau, \boldsymbol{\zeta})$ полностью определяют распределения первого о.п.в. $\mathbf{Z}(t), t \geq 0$, и второго о.п.в. $\mathbf{Y}(t), t \geq 0$. На событии

$$
\left\{T_{k}<t \leq T_{k+1}\right\} \quad \text { при } k \geq 0
$$

выполняются равенства $\nu(t)=k, \eta(t)=k+1, \mathbf{Z}(t)=\mathbf{Z}_{k}, \mathbf{Y}(t)=\mathbf{Z}_{k+1}$. Следовательно, ступенчатые процессы $\nu(t), \mathbf{Z}(t), \eta(t)$ и $\mathbf{Y}(t)$ непрерывны слева при $t>0$. 
Из [15] следует, что предельные теоремы для первого о.п.в. $\mathbf{Z}(t)$ и второго о.п.в. $\mathbf{Y}(t)$ могут существенно различаться.

В настоящей работе, как и в $[8 ; 10 ; 11]$, будем предполагать, что для случайных векторов $\boldsymbol{\xi}_{1}=\left(\tau_{1}, \boldsymbol{\zeta}_{1}\right)$ и $\boldsymbol{\xi}=(\tau, \boldsymbol{\zeta})$, определяющих о.п.в. $\mathbf{Z}(t)$ и $\mathbf{Y}(t)$, выполнено моментное условие Крамера в следующем виде:

$\left[\mathbf{C}_{0}\right] \quad \mathbb{E} e^{\delta\left|\boldsymbol{\xi}_{1}\right|}<\infty, \quad \mathbb{E} e^{\delta|\boldsymbol{\xi}|}<\infty$ при некотором $\delta>0$.

В работах $[8 ; 9 ; 12-14]$, в частности, получены интегро-локальные теоремы для процессов $\mathbf{Z}(t)$ и $\mathbf{Y}(t)$ при $t \rightarrow \infty$ в случае, когда случайный вектор $\boldsymbol{\xi}=(\tau, \boldsymbol{\zeta})$, «определяющий» эти процессы, является нерешетчатым. В настоящей работе (и в одномерном случае для $\mathbf{Z}(t)$ в [11]) решается аналогичная задача для арифметических о.п.в. $\mathbf{Z}(t)$ и $\mathbf{Y}(t)$ (и их непрерывных справа версий), т. е. для случая, когда случайные векторы $\boldsymbol{\xi}_{1}, \boldsymbol{\xi}$ лежат на целочисленной решетке $\mathbb{Z}^{d+1}$. Более точно, мы будем предполагать, что $\mathbb{P}\left(\boldsymbol{\xi}_{1} \in \mathbb{Z}^{d+1}\right)=1$, а для вектора $\boldsymbol{\xi}$ выполнено следующее более сильное условие арифметичности, которое (см. [1, с. 69]) сформулируем в терминах характеристической функции этого вектора:

$$
f(\boldsymbol{u}):=\mathbb{E} e^{i \boldsymbol{u} \boldsymbol{\xi}}, \quad \boldsymbol{u} \in \mathbb{R}^{d+1},
$$

где, напомним, $\boldsymbol{u} \boldsymbol{\xi}$ - скалярное произведение векторов $\boldsymbol{u}$ и $\boldsymbol{\xi}$ в $\mathbb{R}^{d+1}$.

$[\mathbf{Z}]$ (Условие арифметичности) Для любого $\boldsymbol{u} \in \mathbb{Z}^{d+1}$ выполняется равенство $f(2 \pi \boldsymbol{u})=1$ и для любого $\boldsymbol{u} \in \mathbb{R}^{d+1} \backslash \mathbb{Z}^{d+1}-$ неравенство $|f(2 \pi \boldsymbol{u})|<1$.

Заметим попутно, что если для случайного вектора $\boldsymbol{\xi}=(\tau, \boldsymbol{\zeta})$ выполнено условие $[\mathbf{Z}]$, то этот вектор не может быть вырожденным в пространстве $\mathbb{R}^{d+1}$, т. е. с вероятностью 1 лежать на плоскости

$$
L_{\mathbf{n}, c}:=\left\{(u, \mathbf{v}) \in \mathbb{R}^{d+1}: n_{1} u+\mathbf{n}_{2} \mathbf{v}=c\right\},
$$

определяемой единичной нормалью $\mathbf{n}=\left(n_{1}, \mathbf{n}_{2}\right)$ и константой $c$. Действительно, если $\mathbb{P}\left(\boldsymbol{\xi} \in L_{\mathbf{n}, c}\right)=1$, то можно выбрать вещественное $r$ такое, что $\boldsymbol{u}=\left(u_{1}, \boldsymbol{u}_{2}\right):=\left(r n_{1}, r \mathbf{n}_{2}\right) \notin \mathbb{Z}^{d+1}$. Тогда для этого $\boldsymbol{u} \notin \mathbb{Z}^{d+1}$ выполнено равенство

$$
|f(2 \pi \boldsymbol{u})|=\left|\mathbb{E} e^{i 2 \pi r c}\right|=1,
$$

которое в силу условия $[\mathbf{Z}]$ невозможно.

Поскольку арифметические о.п.в. $\mathbf{Z}(t)$ и $\mathbf{Y}(t)$ меняются только в целые моменты времени $t=1,2, \ldots$ и при этом непрерывны слева, то для любого нецелого аргумента $t>0$ выполняются равенства

$$
\mathbf{Z}(t)=\mathbf{Z}([t]+1), \quad \mathbf{Y}(t)=\mathbf{Y}([t]+1),
$$


где, как обычно, через $[t]$ обозначена целая часть неотрицательного числа $t$. Поэтому мы будем рассматривать только целые значения аргумента $t=n \in\{0,1,2, \ldots\}$, т. е. будем изучать случайные последовательности

$$
\{\mathbf{Z}(n)\}_{n \geq 0}, \quad\{\mathbf{Y}(n)\}_{n \geq 0} .
$$

Это несколько упростит обозначения и не приведет к потере общности рассмотрений.

Можно изучать и непрерывные справа версии процессов $\mathbf{Z}(t)$ и $\mathbf{Y}(t)$, положив

$$
\mathbf{Z}_{+}(t):=\mathbf{Z}(t+0), \quad \mathbf{Y}_{+}(t):=\mathbf{Y}(t+0), \quad t \geq 0 .
$$

Иначе говоря, для определения непрерывных справа версий процессов $\mathbf{Z}(t)$ и $\mathbf{Y}(t)$ достаточно рассмотреть непрерывные справа функционалы

$$
\begin{aligned}
& \nu_{+}(t)=\max \left\{k \geq 0: T_{k} \leq t\right\}, \quad t>0, \\
& \eta_{+}(t)=\min \left\{k \geq 0: T_{k}>t\right\},
\end{aligned}
$$

и при $t>0$ положить

$$
\mathbf{Z}_{+}(t):=\mathbf{Z}_{\nu_{+}(t)}, \quad \mathbf{Y}_{+}(t):=\mathbf{Z}_{\eta_{+}(t)} .
$$

Таким образом, наряду с последовательностями (1.4) можно изучать последовательности

$$
\left\{\mathbf{Z}_{+}(n)\right\}_{n \geq 0}, \quad\left\{\mathbf{Y}_{+}(n)\right\}_{n \geq 0} .
$$

Однако изучение последовательностей (1.6) легко сводится к изучению последовательностей (1.4), поскольку (см. определения (1.2), (1.5))

и поэтому

$$
\nu_{+}(n)=\nu(n+1), \quad \eta_{+}(n)=\eta(n+1),
$$

$$
\mathbf{Z}_{+}(n)=\mathbf{Z}(n+1), \quad \mathbf{Y}_{+}(n)=\mathbf{Y}(n+1) .
$$

В настоящей работе получены локальные теоремы для многомерных арифметических о.п.в. $\mathbf{Z}(n), \mathbf{Y}(n)$, в которых найдены точные асимптотики вероятностей

$$
\mathbb{P}(\mathbf{Z}(n)=\boldsymbol{x}), \quad \mathbb{P}(\mathbf{Y}(n)=\boldsymbol{x}), \quad n \rightarrow \infty,
$$

для последовательностей $\boldsymbol{x}=\boldsymbol{x}_{n} \in \mathbb{Z}^{d}$ таких, что точка $\boldsymbol{\alpha}:=\boldsymbol{x} / n$ лежит в некотором фиксированном компакте $K \subset \mathbb{R}^{d}$ (см. теоремы $2.1,2.2$ ниже) либо точка $\boldsymbol{\alpha}$ сходится к некоторому фиксированному $\boldsymbol{\alpha}_{0}$ (см. теоремы $2.1 \mathrm{~A}, 2.2 \mathrm{~A}$ ниже). В силу (1.7) найдены точные асимптотики и для вероятностей (см. следствия 2.1, 2.2 ниже)

$$
\mathbb{P}\left(\mathbf{Z}_{+}(n)=\boldsymbol{x}\right), \quad \mathbb{P}\left(\mathbf{Y}_{+}(n)=\boldsymbol{x}\right), \quad n \rightarrow \infty .
$$


Ранее в [11] аналогичная задача решена для одномерного $(d=1)$ арифметического о.п.в. $\mathbf{Z}(n)$. В основе доказательства локальных теорем для арифметических о.п.в. лежит локальная предельная теорема для функции восстановления

$$
H(t, \boldsymbol{x}):=\sum_{n=0}^{\infty} \mathbb{P}\left(\mathbf{S}_{n}=(t, \boldsymbol{x})\right), \quad(t, \boldsymbol{x}) \in \mathbb{Z}^{d+1},
$$

в которой изучается точная асимптотика $H(t, \boldsymbol{x})$ для последовательностей $(t, \boldsymbol{x})=\left(t_{n}, \boldsymbol{x}_{n}\right) \in \mathbb{Z}^{d+1}$ таких, что точки $(\theta, \boldsymbol{\alpha}):=\frac{1}{n}(t, \boldsymbol{x})$ лежат в некотором фиксированном компакте $K \subset \mathbb{R}^{d+1}$ (теорема 2.3).

Исторические обзоры результатов на близкую тему можно найти в $[1 ; 8]$. По методам настоящая работа следует работам [8; $9 ; 12-14]$, в которых при выполнении моментного условия Крамера $\left[\mathbf{C}_{0}\right]$ получены интегро-локальные предельные теоремы для нерешетчатых о.п.в. $\mathbf{Z}(t), \mathbf{Y}(t)$ и отвечающей им меры восстановления $H(B)$ в одномерном и многомерном случаях соответственно, а также работе [11], в которой получены аналогичные результаты в одномерном случае при выполнении условий $\left[\mathbf{C}_{0}\right]$ и $[\mathbf{Z}]$. Поэтому теоремы 2.1-2.3 настоящей работы дополняют результаты работ $[8 ; 9 ; 11-14]$.

Оставшаяся часть настоящей работы состоит из $\S 2$ и 3. В 2 формулируются основные результаты работы. В этих формулировках будет участвовать ряд «базовых» функций, смысл и свойства которых желательно знать для понимания природы установленных законов, поэтому в 22 мы приведем определения и некоторые свойства этих функций. Подробное их описание можно найти в [8] (одномерный случай) и в [12] (многомерный случай). Доказательства основных утверждений работы размещены в 3.

\section{§2. Формулировки основных утверждений}

2.1. Определения и свойства необходимых функций. В данном параграфе мы кратко приведем определения функций, которые будут играть определяющую роль в локальных предельных теоремах.

Для $(\lambda, \boldsymbol{\mu}) \in \mathbb{R}^{d+1}$ положим

$$
\begin{aligned}
\psi(\lambda, \boldsymbol{\mu}):=\mathbb{E} e^{\lambda \tau+\boldsymbol{\mu} \zeta}, & \psi_{1}(\lambda, \boldsymbol{\mu}):=\mathbb{E} e^{\lambda \tau_{1}+\boldsymbol{\mu} \zeta_{1}} \\
A(\lambda, \boldsymbol{\mu}):=\ln \psi(\lambda, \boldsymbol{\mu}), & A_{1}(\lambda, \boldsymbol{\mu}):=\ln \psi_{1}(\lambda, \boldsymbol{\mu}) \\
\mathcal{A}:=\{(\lambda, \boldsymbol{\mu}): \psi(\lambda, \boldsymbol{\mu})<\infty\}, & \mathcal{A}_{1}:=\left\{(\lambda, \boldsymbol{\mu}): \psi_{1}(\lambda, \boldsymbol{\mu})<\infty\right\} .
\end{aligned}
$$

Ясно, что в соответствии с условием $\left[\mathbf{C}_{0}\right]$ внутренности $(\mathcal{A})$ и $\left(\mathcal{A}_{1}\right)$ множеств $\mathcal{A}$ и $\mathcal{A}_{1}$ содержат точку $(\lambda, \boldsymbol{\mu})=(0, \mathbf{0})$ и являются областями аналитичности функций $\psi(\lambda, \boldsymbol{\mu})$ и $\psi_{1}(\lambda, \boldsymbol{\mu})$ соответственно. 
Ключевую роль в описании асимптотики функции восстановления (см. $(1.8))$, соответствующей случайному блужданию $\left\{\mathbf{S}_{n}\right\}_{n \geq 0}$, играет так называемая вторая функиия уклонений аргументов $\theta \geq 0$ и $\boldsymbol{\alpha} \in \mathbb{R}^{d}$

$$
D(\theta, \boldsymbol{\alpha}):=\sup _{(\lambda, \boldsymbol{\mu}) \in \mathcal{A} \leq 0}\{\lambda \theta+\boldsymbol{\mu} \boldsymbol{\alpha}\}=\sup _{(\lambda, \boldsymbol{\mu}) \in \partial \mathcal{A} \leq 0}\{\lambda \theta+\boldsymbol{\mu} \boldsymbol{\alpha}\},
$$

где

$$
\mathcal{A}^{\leq 0}:=\{(\lambda, \boldsymbol{\mu}): A(\lambda, \boldsymbol{\mu}) \leq 0\}
$$

$\partial \mathcal{A}$ - граница $\mathcal{A}$. Свойства функции $D(\theta, \boldsymbol{\alpha})$ изучены весьма полно (см., например, $[1, \S 2.9 ; 15]$. Она выпукла, полунепрерывна снизу, полуаддитивна, линейна вдоль любого луча, исходящего из точки $\left(\theta_{0}, \boldsymbol{\alpha}_{0}\right)=(0, \mathbf{0})$.

Отметим, что в силу линейности функции $D(\theta, \boldsymbol{\alpha})$ вдоль любого луча, исходящего из точки $(0, \boldsymbol{0})$, при $\theta>0$ выполняется равенство

$$
D(\theta, \boldsymbol{\alpha})=\theta D\left(1, \frac{\boldsymbol{\alpha}}{\theta}\right)
$$

и, стало быть, функция двух переменных $D(\theta, \boldsymbol{\alpha})$ полностью определяется значениями функции одной переменной

$$
D(\boldsymbol{\alpha}):=D(1, \boldsymbol{\alpha}) .
$$

Кроме того, именно в терминах функции $D(\boldsymbol{\alpha})$ будут сформулированы основные результаты настоящей работы. В силу (2.1)

$$
D(\boldsymbol{\alpha})=\sup _{(\lambda, \boldsymbol{\mu}) \in \partial \mathcal{A} \leq 0}\{\boldsymbol{\mu} \boldsymbol{\alpha}+\lambda\}=\sup _{\boldsymbol{\mu}}\{\boldsymbol{\mu} \boldsymbol{\alpha}-A(\boldsymbol{\mu})\},
$$

где

$$
A(\boldsymbol{\mu}):=-\sup \{\lambda: A(\lambda, \boldsymbol{\mu}) \leq 0\}
$$

- это так называемая базовая функиия для о.п.в. В [15] установлено, что функция $A(\boldsymbol{\mu})$ является выпуклой и полунепрерывной снизу и, следовательно (см. [15]), верно равенство

$$
A(\boldsymbol{\mu})=\sup _{\boldsymbol{\alpha}}\{\boldsymbol{\mu} \boldsymbol{\alpha}-D(\boldsymbol{\alpha})\} .
$$

Пусть $(\lambda(\boldsymbol{\alpha}), \boldsymbol{\mu}(\boldsymbol{\alpha}))$ - любая точка, в которой достигается супремум

$$
\sup _{(\lambda, \boldsymbol{\mu}) \in \mathcal{A} \leq 0}\{\lambda+\boldsymbol{\mu} \boldsymbol{\alpha}\}=D(1, \boldsymbol{\alpha})=D(\boldsymbol{\alpha}),
$$

если такая точка существует. В [8] (в одномерном случае) и [12] (в многомерном случае) показано, что функции $D(\boldsymbol{\alpha})$ и $D(\theta, \boldsymbol{\alpha})$ аналитичны в множествах

$$
\mathfrak{A}:=\{\boldsymbol{\alpha}:(\lambda(\boldsymbol{\alpha}), \boldsymbol{\mu}(\boldsymbol{\alpha})) \in(\mathcal{A})\}, \quad \mathcal{D}:=\left\{(\theta, \boldsymbol{\alpha}): \frac{\boldsymbol{\alpha}}{\theta} \in \mathfrak{A}, \theta>0\right\}
$$


соответственно. При этом для $\boldsymbol{\alpha} \in \mathfrak{A}$ точка $(\lambda(\boldsymbol{\alpha}), \boldsymbol{\mu}(\boldsymbol{\alpha}))$ является единственной и выполнены равенства

$$
\begin{aligned}
\lambda(\boldsymbol{\alpha})=-A(\boldsymbol{\mu}(\boldsymbol{\alpha})), & \boldsymbol{\mu}(\boldsymbol{\alpha})=D^{\prime}(\boldsymbol{\alpha}), \\
D(\boldsymbol{\alpha})=\lambda(\boldsymbol{\alpha})+\boldsymbol{\alpha} \boldsymbol{\mu}(\boldsymbol{\alpha}), & A(\lambda(\boldsymbol{\alpha}), \boldsymbol{\mu}(\boldsymbol{\alpha}))=0 .
\end{aligned}
$$

2.2. Локальные теоремы для арифметических обобщенных процессов восстановления. Рассмотрим сначала первый о.п.в.

Асимптотика вероятностей

$$
\mathbb{P}(\mathbf{Z}(n)=\boldsymbol{x})
$$

будет изучаться в зоне нормированных уклонений

$$
\boldsymbol{\alpha}:=\frac{\boldsymbol{x}}{n} \in \mathfrak{A},
$$

и эту зону, по аналогии с областями аналитичности, возникающими в классических предельных теоремах для случайных блужданий, естественно назвать крамеровской зоной. Однако, в отличие от названных классических теорем, асимптотику (2.6) не всегда возможно получить во всей зоне (2.7). В ряде случаев ее приходится сужать. Если $\lambda_{+}>D(\mathbf{0})$, где

$$
\lambda_{+}=\sup \left\{\lambda: \mathbb{E} e^{\lambda \tau}<\infty\right\},
$$

то сужение не требуется.

Рассмотрим случай $\lambda_{+} \leq D(\mathbf{0})$. В этом случае запретной частью зоны $\mathfrak{A}$ будет замкнутое множество

$$
\mathfrak{B}_{Z}:=\left\{\boldsymbol{\alpha} \in \mathbb{R}^{d}: \lambda(\boldsymbol{\alpha}) \geq \lambda_{+}\right\} .
$$

Таким образом, в теореме 2.1 будет изучаться асимптотика вероятностей (2.6) в регулярной зоне уклонений

$$
\boldsymbol{\alpha}:=\frac{\mathbf{x}}{n} \in \mathfrak{A} \backslash \mathfrak{B}_{Z},
$$

а в теореме $2.1^{*}$ - в зоне $(2.7)$.

Обозначим

$$
C(\boldsymbol{\alpha}):=C_{H}(1, \boldsymbol{\alpha}), \quad I_{Z}(\alpha):=\sum_{m=1}^{\infty} e^{\lambda(\alpha) m} \mathbb{P}(\tau \geq m),
$$

где положительная непрерывная в конусе $\mathcal{D}$ функция $C_{H}(\theta, \boldsymbol{\alpha})$ будет определена ниже формулой (3.6). Легко заметить, что для $\boldsymbol{\alpha} \in\left(\mathfrak{B}_{Z}\right)$

$$
I_{Z}(\boldsymbol{\alpha}) \geq \mathbb{E} e^{\lambda(\boldsymbol{\alpha}) \tau}=\infty .
$$

Сформулируем теперь локальную теорему для процесса $\mathbf{Z}(n)$. 
Теорема 2.1. Пусть выполнены условия $\left[\mathbf{C}_{0}\right]$ и $[\mathbf{Z}]$, фиксирован компакт

$$
K \subset \mathfrak{A} \backslash \mathfrak{B}_{Z}
$$

и выполнены условия допустимой неоднородности

$$
\begin{gathered}
\mathcal{A}_{K} \subset\left(\mathcal{A}_{1}\right), \text { где } \mathcal{A}_{K}:=\{(\lambda, \boldsymbol{\mu})=(\lambda(\boldsymbol{\alpha}), \boldsymbol{\mu}(\boldsymbol{\alpha})): \boldsymbol{\alpha} \in K\}, \\
\mathbb{P}\left(\tau_{1} \geq n\right)=o\left(\frac{1}{n^{d / 2}} e^{-n D(\mathbf{0})}\right) \quad \text { при } n \rightarrow \infty, \text { если } \boldsymbol{\alpha}=\mathbf{0} \in K .
\end{gathered}
$$

Тогда при $\boldsymbol{\alpha}:=\boldsymbol{x} / n \in K, \boldsymbol{x} \in \mathbb{Z}^{d}$ имеет место представление

$$
\mathbb{P}(\mathbf{Z}(n)=\boldsymbol{x})=\psi_{1}(\lambda(\boldsymbol{\alpha}), \boldsymbol{\mu}(\boldsymbol{\alpha})) \frac{C(\boldsymbol{\alpha})}{n^{d / 2}} e^{-n D(\boldsymbol{\alpha})} I_{Z}(\boldsymbol{\alpha})(1+o(1)),
$$

в котором остаточный член $о(1)=\varepsilon_{n}(\boldsymbol{x})$ удовлетворяет соотношению

$$
\lim _{n \rightarrow \infty} \sup _{\boldsymbol{x} \in \mathbb{Z}^{d}, \boldsymbol{x} / n \in K}\left|\varepsilon_{n}(\boldsymbol{x})\right|=0 .
$$

Замечание 2.1. Пусть в теореме 2.1 компакт $K$ имеет вид $K=[\boldsymbol{a}]_{\varepsilon}$ для достаточно малого $\varepsilon>0$. Тогда условия допустимой неоднородности (2.9) теоремы 2.1 выполняются автоматически.

В одномерном случае $d=1$ утверждение (2.10) теоремы 2.1 доказано в работе [11]. Теорема 2.1 согласуется с теоремой 1.1 в [8] и с теоремой 3.1 в [12] и дополняет эти теоремы в арифметическом многомерном случае (как уже говорилось, в [8] и [12] получены интегро-локальные теоремы для нерешетчатого случая в одномерном и многомерном случаях соответственно). Как отмечено в [8] и [12], вид суммы $I_{Z}(\boldsymbol{\alpha})$ поясняет в какой-то мере существенность присутствия запретного множества $\mathfrak{B}_{Z}$ : при $\boldsymbol{\alpha} \in\left(\mathfrak{B}_{Z}\right)$ (или, что то же самое, $\left.\lambda(\boldsymbol{\alpha})>\lambda_{+}\right)$сумма $I_{Z}(\boldsymbol{\alpha})$ расходится $($ см. $(2.8))$ и асимптотика последовательности $\mathbb{P}(\mathbf{Z}(n)=\boldsymbol{x})$ будет существенно иной. Существенность условия (2.9) поясняет присутствие в правой части $(2.10)$ множителя $\psi_{1}(\lambda(\boldsymbol{\alpha}), \boldsymbol{\mu}(\boldsymbol{\alpha}))$.

Следствие 2.1. В условиях теоремы 2.1 представление (2.10) сохранится, если в нем $\mathbf{Z}(n)$ заменить на $\mathbf{Z}_{+}(n)$ и $I_{Z}(\boldsymbol{\alpha})-$ на $I_{Z+}(\boldsymbol{\alpha}):=e^{-\lambda(\boldsymbol{\alpha})} I_{Z}(\boldsymbol{\alpha})$.

Обозначим через

$$
\gamma(n):=n-T_{\nu(n)} \quad \text { и } \quad \chi(n):=T_{\nu(n)+1}-n
$$

- величины недоскока до уровня $n$ и перескока через уровень $n$ блуждания $\left\{T_{k}\right\}$ соответственно. Таким образом, слагаемое $\tau_{\nu(n)+1}$, «накрывающее» уровень $n$, представляется в виде суммы $\tau_{\nu(n)+1}=\gamma(n)+\chi(n)$. 
А. А. Боровков обратил внимание авторов на следующий факт: если в локальной теореме для о.п.в. $\mathbf{Z}(n)$ величина недоскока $\gamma(n)$ фиксирована (или ограничена в нужной мере), то можно значительно увеличить зону уклонений, заменив условие $K \subset \mathfrak{A} \backslash \mathfrak{B}_{Z}$ условием $K \subset \mathfrak{A}$. Иначе говоря, справедлива

Теорема 2.1*. Пусть выполнены условия $\left[\mathbf{C}_{0}\right]$ и $[\mathbf{Z}]$, фиксирован компакт

$$
K \subset \mathfrak{A}
$$

и выполнены условия допустимой неоднородности (2.9).

Тогда для любых фиксированных целых $u \geq 1, v \geq 0$, для любой последовательности $\boldsymbol{x}=\boldsymbol{x}_{n} \in \mathbb{Z}^{d}$ такой, что $\boldsymbol{\alpha}:=\boldsymbol{x} / n \in K$, имеют место представления

$$
\begin{aligned}
& \mathbb{P}(\mathbf{Z}(n)=\boldsymbol{x}, \gamma(n)=u) \\
& \quad=\psi_{1}(\lambda(\boldsymbol{\alpha}), \boldsymbol{\mu}(\boldsymbol{\alpha})) \frac{C(\boldsymbol{\alpha})}{n^{d / 2}} e^{-n D(\boldsymbol{\alpha})} I_{Z}(\boldsymbol{\alpha} ; u)\left(1+o_{u}(1)\right), \\
& \mathbb{P}(\mathbf{Z}(n)=\boldsymbol{x}, \gamma(n)=u, \chi(n)=v) \\
& \quad=\psi_{1}(\lambda(\boldsymbol{\alpha}), \boldsymbol{\mu}(\boldsymbol{\alpha})) \frac{C(\boldsymbol{\alpha})}{n^{d / 2}} e^{-n D(\boldsymbol{\alpha})} I_{Z}(\boldsymbol{\alpha} ; u, v)\left(1+o_{u, v}(1)\right),
\end{aligned}
$$

где

$$
I_{Z}(\boldsymbol{\alpha} ; u):=e^{\lambda(\boldsymbol{\alpha}) u} \mathbb{P}(\tau \geq u), \quad I_{Z}(\boldsymbol{\alpha} ; u, v):=e^{\lambda(\boldsymbol{\alpha}) u} \mathbb{P}(\tau=u+v)
$$

и остаточные члены $o_{u}(1)=\varepsilon_{n}(\boldsymbol{x}), o_{u, v}(1)=\varepsilon_{n}(\boldsymbol{x})$ удовлетворяют соотношению (2.11).

Теоремы 2.1, 2.1* будут доказаны в п. 3.3.

Перейдем теперь к формулированию локальной теоремы для второго о.п.в. $\mathbf{Y}(n)$ и его непрерывной справа версии $\mathbf{Y}_{+}(n)$. Обозначим

$$
\mathcal{M}:=\left\{\boldsymbol{\mu} \in \mathbb{R}^{d}: \mathbb{E} e^{\boldsymbol{\mu} \zeta}<\infty\right\} .
$$

В силу условия $\left[\mathbf{C}_{0}\right]$ внутренность $(\mathcal{M})$ содержит точку $\boldsymbol{\mu}=\mathbf{0}$. Для процессов $\mathbf{Y}(n)$ и $\mathbf{Y}_{+}(n)$ запретным множеством будет

$$
\mathfrak{B}_{Y}:=\{\boldsymbol{\alpha} \in \mathfrak{A}: \boldsymbol{\mu}(\boldsymbol{\alpha}) \notin(\mathcal{M})\} .
$$

Обозначим

$$
I_{Y}(\boldsymbol{\alpha}):=\sum_{m=1}^{\infty} e^{\lambda(\boldsymbol{\alpha}) m} \mathbb{E}\left(e^{\boldsymbol{\mu}(\boldsymbol{\alpha}) \boldsymbol{\zeta}} ; \tau \geq m\right) .
$$

Как следует из леммы 3.6 (см. ниже), если $\boldsymbol{\alpha} \in \mathfrak{A} \backslash \mathfrak{B}_{Y}$, mо $I_{Y}(\boldsymbol{\alpha})<\infty$. 
Обратно, если $\boldsymbol{\alpha} \in\left(\mathfrak{B}_{Y}\right)$, то

$$
\begin{aligned}
I_{Y}(\boldsymbol{\alpha}) & :=\sum_{m=1}^{\infty} e^{\lambda(\boldsymbol{\alpha}) m} \mathbb{E}\left(e^{\boldsymbol{\mu}(\boldsymbol{\alpha}) \boldsymbol{\zeta}} ; \tau \geq m\right) \\
& =\sum_{k=1}^{\infty} \mathbb{E}\left(e^{\boldsymbol{\mu}(\boldsymbol{\alpha}) \boldsymbol{\zeta}} ; \tau=k\right) \sum_{m=1}^{k} e^{\lambda(\boldsymbol{\alpha}) m} \\
& \geqslant \sum_{k=1}^{\infty} \mathbb{E}\left(e^{\boldsymbol{\mu}(\boldsymbol{\alpha}) \boldsymbol{\zeta}} ; \tau=k\right) e^{\lambda(\boldsymbol{\alpha})}=e^{\lambda(\boldsymbol{\alpha})} \mathbb{E} e^{\boldsymbol{\mu}(\boldsymbol{\alpha}) \boldsymbol{\zeta}}=\infty
\end{aligned}
$$

и асимптотика вероятности (2.15), как и в случае с процессом $\mathbf{Z}(n)$, в области $\left(\mathfrak{B}_{Y}\right)$ будет существенно другой.

Теорема 2.2. Пусть выполнены условия $\left[\mathbf{C}_{0}\right],[\mathbf{Z}]$ и фиксирован компакт $K$ такой, что

$$
K \subset \mathfrak{A} \backslash \mathfrak{B}_{Y} .
$$

Пусть для начального случайного вектора $\boldsymbol{\xi}_{1}=\left(\tau_{1}, \boldsymbol{\zeta}_{1}\right)$ выполнено условие допустимой неоднородности

$$
(0, \boldsymbol{\mu}(\boldsymbol{\beta})) \in\left(\mathcal{A}_{1}\right) \text { и }(\lambda(\boldsymbol{\beta}), \boldsymbol{\mu}(\boldsymbol{\beta})) \in\left(\mathcal{A}_{1}\right) \text { при всех } \boldsymbol{\beta} \in K .
$$

Тогда для $\boldsymbol{\alpha}:=\boldsymbol{x} / n \in K, \boldsymbol{x} \in \mathbb{Z}^{d}$ при $n \rightarrow \infty$ имеет место соотношение

$$
\mathbb{P}(\mathbf{Y}(n)=\boldsymbol{x})=\psi_{1}(\lambda(\boldsymbol{\alpha}), \boldsymbol{\mu}(\boldsymbol{\alpha})) \frac{C(\boldsymbol{\alpha})}{n^{d / 2}} e^{-n D(\boldsymbol{\alpha})} I_{Y}(\boldsymbol{\alpha})(1+o(1)),
$$

в котором остаточный член о $(1)=\varepsilon_{n}(\boldsymbol{x})$ удовлетворяет соотношению (2.11).

Замечание 2.2. Пусть в теореме 2.2 компакт $K$ имеет вид $K=[\boldsymbol{a}]_{\varepsilon}$ для достаточно малого $\varepsilon>0$. Тогда условие допустимой неоднородности (2.14) теоремы 2.2 выполняется автоматически.

Следствие 2.2. При выполнении условий теоремы 2.2 представление (2.15) сохранится, если в нем $\mathbf{Y}(n)$ заменить на $\mathbf{Y}_{+}(n)$ и $I_{Y}(\boldsymbol{\alpha})-$ на $I_{Y+}(\boldsymbol{\alpha}):=e^{-\lambda(\boldsymbol{\alpha})} I_{Y}(\boldsymbol{\alpha})$.

Если в локальной теореме для о.п.в. $\mathbf{Y}(n)$ фиксировать величину перескока $\chi(n)$, то можно значительно увеличить зону уклонений, заменив условие $K \subset \mathfrak{A} \backslash \mathfrak{B}_{Y}(3.21)$ условием $K \subset \mathfrak{A}$. Иначе говоря, справедлива

Теорема 2.2*. Пусть выполнены условия $\left[\mathbf{C}_{0}\right],[\mathbf{Z}]$, фиксирован компакт $K$ такой, что

$$
K \subset \mathfrak{A}
$$

и выполнено условие допустимой неоднородности (2.14). 
Тогда для любого фиксированного целого $v \geq 0$, для любой последовательности $\boldsymbol{x}=\boldsymbol{x}_{n} \in \mathbb{Z}^{d}$ такой, что $\boldsymbol{\alpha}:=\frac{\boldsymbol{x}}{n} \in K$, имеет место представление

$$
\begin{aligned}
& \mathbb{P}(\mathbf{Y}(n)=\boldsymbol{x}, \chi(n)=v) \\
& \quad=\psi_{1}(\lambda(\boldsymbol{\alpha}), \boldsymbol{\mu}(\boldsymbol{\alpha})) \frac{C(\boldsymbol{\alpha})}{n^{d / 2}} e^{-n D(\boldsymbol{\alpha})} I_{Y}(\boldsymbol{\alpha} ; v)\left(1+o_{v}(1)\right),
\end{aligned}
$$

где

$$
I_{Y}(\boldsymbol{\alpha} ; v):=e^{-\lambda(\boldsymbol{\alpha}) v}
$$

и остаточный член $o_{w}(1)=\varepsilon_{n}(\boldsymbol{x})$ удовлетворяет соотношению (2.11).

Теоремы 2.2 и $2.2^{*}$ будут доказаны в п. 3.4 .

Заметим попутно, что доказательства теорем 2.1 и 2.2 в ключевых местах существенно различаются: в первом доказательстве приходится бороться с «толстым правым хвостом» случайной величины $\tau$, а во втором с «толстыми хвостами» случайного вектора $\boldsymbol{\zeta}$. Поэтому мы не можем обойтись теоремой 2.1 только для одной версии обобщенного процесса восстановления, получив необходимое утверждение для второй версии в качестве простого следствия.

Обратимся теперь к умеренно большим и нормальным уклонениям для о.п.в.

Для $\boldsymbol{\alpha} \in \mathbb{R}^{d}, \varepsilon>0$ обозначим через

$$
[\boldsymbol{\alpha}]_{\varepsilon}:=\left\{\boldsymbol{\beta} \in \mathbb{R}^{d}:|\boldsymbol{\beta}-\boldsymbol{\alpha}| \leq \varepsilon\right\}
$$

замкнутый шар радиуса $\varepsilon$ с центром в $\boldsymbol{\alpha}$. Положим

$$
a:=\frac{\mathbb{E} \zeta}{\mathbb{E} \tau} .
$$

Рассмотрим теперь более подробно локальные теоремы для процессов $\mathbf{Z}(n)$ и $\mathbf{Y}(n)$ в областях умеренно больших и нормальных уклонений. Обозначим

$$
B^{2}:=\frac{1}{\mathbb{E} \tau} B_{0}^{2}, \quad \sigma^{2}:=\left|B^{2}\right|,
$$

где

$$
B_{0}^{2}:=\mathbb{E}(\boldsymbol{\zeta}-\boldsymbol{a} \tau)(\boldsymbol{\zeta}-\boldsymbol{a} \tau)^{\top}
$$

- ковариационная матрица случайного вектора $\boldsymbol{\zeta}-\boldsymbol{a} \tau, \boldsymbol{\top}-$ знак транспонирования.

Лемма 2.1. Пусть выполнено условие $\left[\mathbf{C}_{0}\right]$. Тогда выполнены равенства

$$
\begin{gathered}
C(\boldsymbol{a}) I_{Z}(\boldsymbol{a})=C(\boldsymbol{a}) I_{Y}(\boldsymbol{a})=\frac{1}{\sigma(2 \pi)^{d / 2}} \\
D(\boldsymbol{a})=0, \quad D^{\prime}(\boldsymbol{a})=\mathbf{0}, \quad D^{\prime \prime}(\boldsymbol{a})=B^{-2}
\end{gathered}
$$


Лемму 2.1 докажем в п. 3.5. Из теорем 2.1, 2.2 и леммы 2.1 с учетом замечаний 2.1 и 2.2 вытекает

Следствие 2.3. Пусть выполнены условия $\left[\mathbf{C}_{0}\right],[\mathbf{Z}]$. Тогда для любой последовательности $\boldsymbol{x}=\boldsymbol{x}_{n} \in \mathbb{Z}^{d}$ такой, что $\boldsymbol{\alpha}:=\frac{\boldsymbol{x}}{n} \rightarrow \boldsymbol{a}$ при $n \rightarrow \infty$, имеют место соотношения

$$
\mathbb{P}(\mathbf{Z}(n)=\boldsymbol{x}) \sim \mathbb{P}(\mathbf{Y}(n)=\boldsymbol{x}) \sim \frac{1}{\sigma(2 \pi n)^{d / 2}} e^{-n D(\boldsymbol{\alpha})},
$$

$B$ частности, если $\boldsymbol{\alpha}-\boldsymbol{a}=o\left(\frac{1}{n^{1 / 3}}\right)$ при $n \rightarrow \infty$, то для $\boldsymbol{y}:=\boldsymbol{x}-n \boldsymbol{a}$ справедливо соотношение

откуда следует, что

$$
n D(\boldsymbol{\alpha})=\frac{\boldsymbol{y} B^{-2} \boldsymbol{y}^{\top}}{2 n}+o(1)
$$

$$
\mathbb{P}(\mathbf{Z}(n)=\boldsymbol{x}) \sim \mathbb{P}(\mathbf{Y}(n)=\boldsymbol{x}) \sim \frac{1}{\sigma(2 \pi n)^{d / 2}} \exp \left\{-\frac{\boldsymbol{y} B^{-2} \boldsymbol{y}^{\top}}{2 n}\right\} .
$$

Таким образом, локальные теоремы для о.п.в. $\mathbf{Z}(n)$ и $\mathbf{Y}(n)$ в областях умеренно больших и нормальных уклонений совпадают.

2.3. Локальная теорема для функиии восстановления. В арифметическом случае для доказательства теорем 2.1, 2.2 существенную роль играет локальная предельная теорема 2.3 для функции восстановления $($ см. $(1.8))$, в которой для последовательности $(t, \boldsymbol{x})=\left(t_{n}, \boldsymbol{x}_{n}\right) \in \mathbb{Z}^{d+1}$ изучается асимптотика $H(t, \boldsymbol{x})$ при $n \rightarrow \infty$ в случае, когда точка $(\theta, \boldsymbol{\alpha}):=$ $\frac{1}{n}(t, \boldsymbol{x})$ лежит в некотором фиксированном компакте $K$, вложенном в область $\mathcal{D}$ аналитичности функции $D(\theta, \boldsymbol{\alpha})$.

Теорема 2.3. Пусть выполнены условия $\left[\mathbf{C}_{0}\right]$ и $[\mathbf{Z}]$ и для фиксированного компакта

$$
K \subset \mathcal{D}
$$

выполнено условие допустимой неоднородности

$$
\mathcal{A}_{K} \subset\left(\mathcal{A}_{1}\right), \text { где } \mathcal{A}_{K}:=\left\{(\lambda, \boldsymbol{\mu})=\left(\lambda\left(\frac{\boldsymbol{\alpha}}{\theta}\right), \boldsymbol{\mu}\left(\frac{\boldsymbol{\alpha}}{\theta}\right)\right):(\theta, \boldsymbol{\alpha}) \in K\right\} .
$$

Тогда для $(t, \boldsymbol{x}) \in \mathbb{Z}^{d+1},(\theta, \boldsymbol{\alpha}):=\frac{1}{n}(t, \boldsymbol{x})$ имеет место представление

$$
H(t, \boldsymbol{x})=\psi_{1}\left(\lambda\left(\frac{\boldsymbol{\alpha}}{\theta}\right), \boldsymbol{\mu}\left(\frac{\boldsymbol{\alpha}}{\theta}\right)\right) \frac{C_{H}(\theta, \boldsymbol{\alpha})}{n^{d / 2}} e^{-n D(\theta, \boldsymbol{\alpha})}(1+o(1)),
$$

в котором остаточный член $о(1)=\varepsilon_{n}(t, \boldsymbol{x})$ удовлетворяет соотношению

$$
\lim _{n \rightarrow \infty} \sup _{\substack{(t, \boldsymbol{x}) \in \mathbb{Z}^{d+1} \\(t / n, \boldsymbol{x} / n) \in K}}\left|\varepsilon_{n}(t, \boldsymbol{x})\right|=0,
$$

функция $C_{H}(\theta, \boldsymbol{\alpha})$ определена формулой (3.6). 
В одномерном случае $d=1$ теорема 2.3 доказана в [11]; она дополняет теорему 4.1 в [9] и теорему 4.1 в [12], где установлены интегро-локальные теоремы для функции восстановления в нерешетчатом одномерном и многомерном случаях соответственно.

\section{§3. Доказательства основных утверждений}

3.1. Первая функиия уклонений. Локальная теорема для однородного случайного блуждания. В доказательствах основных утверждений настоящей работы (как и в $[8 ; 9 ; 12])$ используются известные интегролокальные теоремы для сумм $\mathbf{S}_{n}=\left(T_{n}, \mathbf{Z}_{n}\right)$ (см., например, $[1, \S 2.9]$ или теорему 3.1 ниже). Важную роль при этом играет (первая) функиия уклонений

$$
\Lambda(\theta, \boldsymbol{\alpha}):=\sup _{(\lambda, \boldsymbol{\mu})}\{\lambda \theta+\boldsymbol{\mu} \boldsymbol{\alpha}-A(\lambda, \boldsymbol{\mu})\},
$$

соответствующая случайному вектору $\boldsymbol{\xi}=(\tau, \boldsymbol{\zeta})$. Это есть преобразование Лежандра над выпуклой полунепрерывной снизу функцией $A(\lambda, \boldsymbol{\mu})$; поэтому функция $\Lambda(\theta, \boldsymbol{\alpha})$ также выпукла и полунепрерывна снизу.

Условимся о следующих обозначениях. Если дана функция $F(u, \boldsymbol{v})$ двух переменных $u \in \mathbb{R}$ и $\boldsymbol{v} \in \mathbb{R}^{d}$, то в дальнейшем нижними индексами (1) и (2) мы будем отмечать производные по первому и второму (градиент) аргументу соответственно, например:

$$
\begin{aligned}
& F_{(1)}^{\prime}\left(u_{1}, \boldsymbol{v}_{1}\right)=\left.\frac{\partial}{\partial u} F\left(u, \boldsymbol{v}_{1}\right)\right|_{u=u_{1}}, \quad F_{(1)}^{\prime \prime}\left(u_{1}, \boldsymbol{v}_{1}\right)=\left.\frac{\partial^{2}}{\partial u^{2}} F\left(u, \boldsymbol{v}_{1}\right)\right|_{u=u_{1}}, \\
& F_{(2)}^{\prime \prime}\left(u_{1}, \boldsymbol{v}_{1}\right)=\left.\frac{\partial^{2}}{\partial \boldsymbol{v}^{2}} F\left(u_{1}, \boldsymbol{v}\right)\right|_{\boldsymbol{v}=\boldsymbol{v}_{1}}, \quad F_{(2,1)}^{\prime \prime}\left(u_{1}, \boldsymbol{v}_{1}\right)=\left.\frac{\partial}{\partial u} \frac{\partial}{\partial \boldsymbol{v}} F(u, \boldsymbol{v})\right|_{(u, \boldsymbol{v})=\left(u_{1}, \boldsymbol{v}_{1}\right)} .
\end{aligned}
$$

Через $F^{\prime}=F^{\prime}(u, \boldsymbol{v})$ и $F^{\prime \prime}=F^{\prime \prime}(u, \boldsymbol{v})$ мы будем обозначать вектор

$$
F^{\prime}=F^{\prime}(u, \boldsymbol{v})=\left(F_{(1)}^{\prime}(u, \boldsymbol{v}), F_{(2)}^{\prime}(u, \boldsymbol{v})\right)
$$

первых производных и матрицу

$$
F^{\prime \prime}=F^{\prime \prime}(u, \boldsymbol{v})
$$

порядка $d+1$ вторых производных соответственно. Через $\left|F^{\prime \prime}\right|$ обозначим определитель матрицы $F^{\prime \prime}$.

Наряду с множествами $\mathcal{A}_{1}$ и $\mathcal{A}$ нам понадобится область $\mathcal{L}$ аналитичности функции $\Lambda(\theta, \boldsymbol{\alpha})$. Эта область содержит те точки $(\theta, \boldsymbol{\alpha}) \in \mathbb{R}^{d+1}$, для которых система уравнений

$$
\left\{\begin{array}{l}
A_{(1)}^{\prime}(\lambda, \boldsymbol{\mu})=\theta, \\
A_{(2)}^{\prime}(\lambda, \boldsymbol{\mu})=\boldsymbol{\alpha}
\end{array}\right.
$$


для координат точки $(\lambda, \boldsymbol{\mu})$ (где достигается верхняя грань в $(3.1))$ имеет решение

$$
(\lambda(\theta, \boldsymbol{\alpha}), \boldsymbol{\mu}(\theta, \boldsymbol{\alpha}))
$$

принадлежащее $(\mathcal{A})$, так что

$$
\mathcal{L}=\left\{A^{\prime}(\lambda, \boldsymbol{\mu}):(\lambda, \boldsymbol{\mu}) \in(\mathcal{A})\right\} .
$$

Так как функция $A(\lambda, \boldsymbol{\mu})$ строго выпукла в $(\mathcal{A})$, такое решение всегда единственно. Сказанное означает, что условия

$$
(\theta, \boldsymbol{\alpha}) \in \mathcal{L} \text { и }(\lambda(\theta, \boldsymbol{\alpha}), \boldsymbol{\mu}(\theta, \boldsymbol{\alpha})) \in(\mathcal{A})
$$

эквивалентны. При этом отображения

$$
(\theta, \boldsymbol{\alpha})=A^{\prime}(\lambda, \boldsymbol{\mu}) \text { и }(\lambda, \boldsymbol{\mu})=\Lambda^{\prime}(\theta, \boldsymbol{\alpha})
$$

взаимно обратны; они осуществляют взаимно однозначное отображение между областями $\mathcal{A}$ и $\mathcal{L}$. Ясно, что точка $(\theta, \boldsymbol{\alpha})=\left(a_{\tau}, \boldsymbol{a}_{\boldsymbol{\zeta}}\right)$, где $a_{\tau}:=\mathbb{E} \tau$, $\boldsymbol{a}_{\boldsymbol{\zeta}}:=\mathbb{E} \boldsymbol{\zeta}$, всегда принадлежит $\mathcal{L} ;$ для нее

$$
\left(\lambda\left(a_{\tau}, \boldsymbol{a}_{\boldsymbol{\zeta}}\right), \boldsymbol{\mu}\left(a_{\tau}, \boldsymbol{a}_{\boldsymbol{\zeta}}\right)\right)=(0, \mathbf{0}) \in(\mathcal{A}) .
$$

Известно (см., например, $[1 ; 8 ; 12])$, что при $(\theta, \boldsymbol{\alpha}) \in \mathcal{L}$ выполняется

$$
\Lambda^{\prime}(\theta, \boldsymbol{\alpha})=\left(\Lambda_{(1)}^{\prime}(\theta, \boldsymbol{\alpha}), \Lambda_{(2)}^{\prime}(\theta, \boldsymbol{\alpha})\right)=(\lambda(\theta, \boldsymbol{\alpha}), \boldsymbol{\mu}(\theta, \boldsymbol{\alpha})),
$$

где пара функций $\lambda(\theta, \boldsymbol{\alpha}), \boldsymbol{\mu}(\theta, \boldsymbol{\alpha})$ является единственным решением системы (3.2).

Из теоремы 2.3.2 в [1, с. 72] вытекает следующая версия локальной теоремы для сумм $\mathbf{S}_{n}$ случайных векторов в арифметическом случае.

Теорема 3.1. Для $n \geq 1$ пусть

$$
\mathbf{S}_{n}:=\sum_{k=1}^{n} \boldsymbol{\xi}_{k}=\left(T_{n}, \mathbf{Z}_{n}\right)
$$

- сумма независимых одинаково распределенных случайных векторов. Пусть выполнены условия $\left[\mathbf{C}_{0}\right]$ и $[\mathbf{Z}]$, компакт $K \subset \mathcal{L}$ фиксирован и точка $(t, \boldsymbol{x}) \in \mathbb{Z}^{d+1}$ такова, что $(\gamma, \boldsymbol{\beta}):=(t / n, \boldsymbol{x} / n) \in K$. Тогда для

$$
C_{1}(\gamma, \boldsymbol{\beta}):=\frac{\sqrt{\left|\Lambda^{\prime \prime}(\gamma, \boldsymbol{\beta})\right|}}{(2 \pi)^{(d+1) / 2}}
$$

при $n \rightarrow \infty$ выполняется

$$
\mathbb{P}\left(T_{n}=t, \mathbf{Z}_{n}=\boldsymbol{x}\right)=\frac{C_{1}(\gamma, \boldsymbol{\beta})}{n^{(d+1) / 2}} e^{-n \Lambda(\gamma, \boldsymbol{\beta})}(1+o(1)),
$$

где остаточный член $о(1)=\varepsilon_{n}(t, \boldsymbol{x})$ равномерен по $(\gamma, \boldsymbol{\beta}) \in K$ :

$$
\lim _{n \rightarrow \infty} \sup _{\substack{(t, \boldsymbol{x}) \in \mathbb{Z}^{d+1},(t / n, \boldsymbol{x} / n) \in K}}\left|\varepsilon_{n}(t, \boldsymbol{x})\right|=0 .
$$


3.2. Доказательство теоремы 2.3. Приведем сначала эквивалентную теореме 2.3 форму локальной теоремы для функции восстановления (которая более удобна для доказательства).

Теорема 2.3A. Пусть выполнены условия $\left[\mathbf{C}_{0}\right]$ и $[\mathbf{Z}]$ и для фиксированной точки

$$
\left(\theta_{0}, \boldsymbol{\alpha}_{0}\right) \in \mathcal{D}
$$

выполнено условие допустимой неоднородности

$$
\left(\lambda\left(\frac{\boldsymbol{\alpha}_{0}}{\theta_{0}}\right), \boldsymbol{\mu}\left(\frac{\boldsymbol{\alpha}_{0}}{\theta_{0}}\right)\right) \in\left(\mathcal{A}_{1}\right) .
$$

Тогда для любой последовательности

$$
(t, \boldsymbol{x}) \in \mathbb{Z}^{d+1}
$$

такой, что

$$
(\theta, \boldsymbol{\alpha}):=\left(\frac{t}{n}, \frac{\boldsymbol{x}}{n}\right) \rightarrow\left(\theta_{0}, \boldsymbol{\alpha}_{0}\right) \text { при } n \rightarrow \infty,
$$

имеет место представление (2.19), в котором остаточный член o(1) $=\varepsilon_{n}$ удовлетворяет соотношению

$$
\lim _{n \rightarrow \infty}\left|\varepsilon_{n}\right|=0
$$

В работе [11] приведено доказательство эквивалентности теорем 2.3 и $2.3 \mathrm{~A}$ в одномерном случае $d=1$. Это доказательство сохраняется и для случая $d \geq 1$.

При $(\theta, \boldsymbol{\alpha}) \in \mathcal{D}$ положим для краткости

$$
\begin{gathered}
(\widehat{\theta}, \widehat{\boldsymbol{\alpha}}):=A^{\prime}(\widehat{\lambda}, \widehat{\boldsymbol{\mu}}), \\
(\widehat{\lambda}, \widehat{\boldsymbol{\mu}}):=\left(\lambda\left(\frac{\boldsymbol{\alpha}}{\theta}\right), \mu\left(\frac{\alpha}{\theta}\right)\right)=D^{\prime}(\theta, \boldsymbol{\alpha})=\left(-A\left(\boldsymbol{\mu}\left(\frac{\boldsymbol{\alpha}}{\theta}\right)\right), \boldsymbol{\mu}\left(\frac{\boldsymbol{\alpha}}{\theta}\right)\right),
\end{gathered}
$$

так что векторы

$$
\begin{aligned}
& (\widehat{\lambda}, \widehat{\boldsymbol{\mu}})=(\widehat{\lambda}(\theta, \boldsymbol{\alpha}), \widehat{\boldsymbol{\mu}}(\theta, \boldsymbol{\alpha}))=\left(\lambda\left(\frac{\boldsymbol{\alpha}}{\theta}\right), \boldsymbol{\mu}\left(\frac{\boldsymbol{\alpha}}{\theta}\right)\right) \\
& (\widehat{\theta}, \widehat{\boldsymbol{\alpha}})=(\widehat{\theta}(\theta, \boldsymbol{\alpha}), \widehat{\boldsymbol{\alpha}}(\theta, \boldsymbol{\alpha}))=A^{\prime}\left(\lambda\left(\frac{\boldsymbol{\alpha}}{\theta}\right), \boldsymbol{\mu}\left(\frac{\boldsymbol{\alpha}}{\theta}\right)\right)
\end{aligned}
$$

суть функции одной переменной $\boldsymbol{\alpha} / \theta$.

Для доказательства теоремы 2.3А нам также понадобится 
Лемма 3.1 [8; 9]. Пусть выполнено условие $\left[\mathbf{C}_{0}\right]$, и пусть $(\theta, \boldsymbol{\alpha}) \in \mathcal{D}$. Тогда справедливы следующие утверждения.

I. Минимум функции $L(r)=L_{\theta, \boldsymbol{\alpha}}(r):=r \Lambda(\theta / r, \boldsymbol{\alpha} / r)$ достигается в единственной точке

$$
r_{\theta, \boldsymbol{\alpha}}=\frac{\theta}{\widehat{\theta}}, \text { и при этом } r_{\theta, \boldsymbol{\alpha}} \widehat{\boldsymbol{\alpha}}=\boldsymbol{\alpha} .
$$

Для функций $\widehat{\lambda}$ и $\widehat{\boldsymbol{\mu}}$ справедливы представления

$$
\widehat{\lambda}=\lambda\left(\frac{\theta}{r_{\theta, \boldsymbol{\alpha}}}, \frac{\boldsymbol{\alpha}}{r_{\theta, \boldsymbol{\alpha}}}\right), \quad \widehat{\boldsymbol{\mu}}=\boldsymbol{\mu}\left(\frac{\theta}{r_{\theta, \boldsymbol{\alpha}}}, \frac{\boldsymbol{\alpha}}{r_{\theta, \boldsymbol{\alpha}}}\right),
$$

где функции $\lambda(\cdot, \cdot)$ и $\boldsymbol{\mu}(\cdot, \cdot)$ являются решением системы (3.2). При этом

$$
L^{\prime}\left(r_{\theta, \boldsymbol{\alpha}}\right)=0, \quad L^{\prime \prime}\left(r_{\theta, \boldsymbol{\alpha}}\right)=\frac{1}{r_{\theta, \boldsymbol{\alpha}}}(\widehat{\theta}, \widehat{\boldsymbol{\alpha}}) \widehat{\Lambda}^{\prime \prime}(\widehat{\theta}, \widehat{\boldsymbol{\alpha}})^{\top}>0
$$

где функции $\widehat{\theta}=\widehat{\theta}(\theta, \boldsymbol{\alpha}), \widehat{\boldsymbol{\alpha}}=\widehat{\boldsymbol{\alpha}}(\theta, \boldsymbol{\alpha})$ определены в $(3.4),(3.5)$,

$$
\widehat{\Lambda}^{\prime \prime}:=\left.\Lambda^{\prime \prime}(\theta, \boldsymbol{\alpha})\right|_{(\theta, \boldsymbol{\alpha})=(\widehat{\theta}, \widehat{\boldsymbol{\alpha}})} .
$$

II. Для заданного $\varepsilon>0$ найдется $\varepsilon_{1}>0$ такое, что

$$
\min _{\left|r-r_{\theta, \boldsymbol{\alpha}}\right| \geq \varepsilon} L(r) \geq L\left(r_{\theta, \boldsymbol{\alpha}}\right)+\varepsilon_{1}
$$

Для $(\theta, \boldsymbol{\alpha}) \in \mathcal{D}$ положим

$$
C_{H}(\theta, \boldsymbol{\alpha}):=\sqrt{\frac{\widehat{\theta}^{d}\left|\Lambda^{\prime \prime}(\widehat{\theta}, \widehat{\boldsymbol{\alpha}})\right|}{(2 \pi \theta)^{d} Q(\widehat{\theta}, \widehat{\boldsymbol{\alpha}})}}, \quad Q(\widehat{\theta}, \widehat{\boldsymbol{\alpha}}):=(\widehat{\theta}, \widehat{\boldsymbol{\alpha}}) \Lambda^{\prime \prime}(\widehat{\theta}, \widehat{\boldsymbol{\alpha}})(\widehat{\theta}, \widehat{\boldsymbol{\alpha}})^{\top},
$$

так что

$$
(\widehat{\theta}, \widehat{\boldsymbol{\alpha}}) \Lambda^{\prime \prime}(\widehat{\theta}, \widehat{\boldsymbol{\alpha}})(\widehat{\theta}, \widehat{\boldsymbol{\alpha}})^{\top}:=\widehat{\theta}^{2} \Lambda_{(1)}^{\prime \prime}(\widehat{\theta}, \widehat{\boldsymbol{\alpha}})+2 \widehat{\theta} \Lambda_{(1,2)}^{\prime \prime}(\widehat{\theta}, \widehat{\boldsymbol{\alpha}}) \widehat{\boldsymbol{\alpha}}^{\top}+\widehat{\boldsymbol{\alpha}} \Lambda_{(2)}^{\prime \prime}(\widehat{\theta}, \widehat{\boldsymbol{\alpha}}) \widehat{\boldsymbol{\alpha}}^{\top}
$$

Доказательство теоремы 2.3А. Опираясь на теорему 3.1 и лемму 3.1, осуществим доказательство в два этапа.

I. Меру восстановления для однородного случая, когда векторы $\boldsymbol{\xi}_{1}=$ $\left(\tau_{1}, \boldsymbol{\zeta}_{1}\right)$ и $\boldsymbol{\xi}=(\tau, \boldsymbol{\zeta})$ (т. е. все слагаемые $\boldsymbol{\xi}_{i}$ равны $\left(\tau_{i}, \boldsymbol{\zeta}_{i}\right)$ при $\left.i \geq 1\right)$ имеют одинаковое распределение, обозначим через $H_{0}(B)$. На этапе I установим утверждение теоремы $2.3 \mathrm{~A}$ для однородного случая. Достаточно доказать, что для любого фиксированного $\left(\theta_{0}, \boldsymbol{\alpha}_{0}\right) \in \mathcal{D}$ и любой последовательности 
$(t, \boldsymbol{x}) \in \mathbb{Z}^{d+1}$ такой, что $(\theta, \boldsymbol{\alpha}):=\frac{1}{n}(t, \boldsymbol{x}) \rightarrow\left(\theta_{0}, \boldsymbol{\alpha}_{0}\right)$ при $n \rightarrow \infty$, имеет место соотношение

$$
H_{0}(\{t\} \times\{\boldsymbol{x}\})=\frac{1}{n^{d / 2}} C_{H}(\theta, \boldsymbol{\alpha}) e^{-n D(\theta, \boldsymbol{\alpha})}(1+o(1)),
$$

совпадающее с соотношением (2.19) при $\psi_{1}=\psi$. Доказательство (3.7) на базе теоремы 3.1 и леммы 3.1 является повторением доказательства интегро-локальной теоремы для функции восстановления в однородном случае в работе [12] (см. также доказательство локальной теоремы в одномерном арифметическом случае в [11]), поэтому мы его опускаем.

II. На этом этапе меру восстановления в неоднородном случае представим в виде

$$
H(t, \boldsymbol{x})=\mathbb{E} H_{0}\left(\left\{t-\tau_{1}\right\} \times\left\{\boldsymbol{x}-\boldsymbol{\zeta}_{1}\right\}\right)
$$

и воспользуемся нижеследующим утверждением (ср. с леммой 4.2 в [9], которая установлена при доказательстве теоремы 3.1 в [9]).

Лемма 3.2. Пусть выполнены условия теоремы $2.3 \mathrm{~A}$. Тогда для некоторых $c>0, C<\infty, n_{0}<\infty$ и при всех $n \geq n_{0}$ имеем

$$
\left|H(t, \boldsymbol{x})-\mathbb{E}\left(H_{0}\left(\left\{t-\tau_{1}\right\} \times\left\{\boldsymbol{x}-\boldsymbol{\zeta}_{1}\right\}\right) ;\left|\boldsymbol{\xi}_{1}\right| \leq \ln ^{2} n\right)\right| \leq C e^{-n D(\theta, \boldsymbol{\alpha})-c \ln ^{2} n} .
$$

Поскольку доказательство леммы 3.2 повторяет доказательство леммы 4.2 из [9] (см. также доказательства леммы 4.1 в [12] и леммы 3.2 в [11]), мы его опускаем.

В силу (3.8) для доказательства утверждения (2.19) в общем случае достаточно отыскать асимптотику среднего

$$
\mathbb{E}\left(H_{0}\left(\left\{t-\tau_{1}\right\} \times\left\{\boldsymbol{x}-\boldsymbol{\zeta}_{1}\right\}\right) ;\left|\boldsymbol{\xi}_{1}\right| \leq \ln ^{2} n\right) .
$$

На основании результата этапа I и (3.8) имеем

$$
\begin{gathered}
H(t, \boldsymbol{x})=\frac{1}{n^{d / 2}} \mathbb{E}\left(C_{1}\left(\theta-\frac{\tau_{1}}{n}, \boldsymbol{\alpha}-\frac{\boldsymbol{\zeta}_{1}}{n}\right) e^{-n D\left(\theta-\tau_{1} / n, \boldsymbol{\alpha}-\boldsymbol{\zeta}_{1} / n\right)}(1+o(1)) ;\left|\boldsymbol{\xi}_{1}\right| \leq \ln ^{2} n\right) \\
+o\left(\frac{1}{n^{d / 2}} e^{-n D(\theta, \boldsymbol{\alpha})}\right) .
\end{gathered}
$$

Учитывая, что в области $\left|\boldsymbol{\xi}_{1}\right| \leq \ln ^{2} n$ при $n \rightarrow \infty$ выполняется

$$
-n D\left(\theta-\frac{\tau_{1}}{n}, \boldsymbol{\alpha}-\frac{\boldsymbol{\zeta}_{1}}{n}\right)=-n D(\theta, \boldsymbol{\alpha})+\widehat{\lambda} \tau_{1}+\widehat{\boldsymbol{\mu}} \boldsymbol{\zeta}_{1}+o(1)
$$

заключаем, что правая часть (3.9) совпадает с правой частью (2.19). 
3.3. Доказательство теорем 2.1, 2.1* и следствия 2.1. Приведем теперь другую (эквивалентную) форму теоремы 2.1, которая более удобна для доказательства (но менее удобна для применения).

Теорема 2.1 А. Пусть выполнены условия $\left[\mathbf{C}_{0}\right]$ и $[\mathbf{Z}]$ и для фиксированной точки

$$
\boldsymbol{\alpha}_{0} \in \mathfrak{A} \backslash \mathfrak{B}_{Z}
$$

выполнены условия допустимой неоднородности

$$
\begin{gathered}
\left(\lambda\left(\boldsymbol{\alpha}_{0}\right), \boldsymbol{\mu}\left(\boldsymbol{\alpha}_{0}\right)\right) \in\left(\mathcal{A}_{1}\right), \\
\mathbb{P}\left(\tau_{1} \geq n\right)=o\left(\frac{1}{n^{d / 2}} e^{-n D(\mathbf{0})}\right) \text { при } n \rightarrow \infty, \text { если } \boldsymbol{\alpha}_{0}=\mathbf{0} .
\end{gathered}
$$

Тогда для любой последовательности $\boldsymbol{x}=\boldsymbol{x}_{n} \in \mathbb{Z}^{d}$ такой, что

$$
\lim _{n \rightarrow \infty} \boldsymbol{\alpha}=\boldsymbol{\alpha}_{0}, \quad \text { где } \boldsymbol{\alpha}:=\frac{\boldsymbol{x}}{n},
$$

имеет место представление $(2.10)$, в котором остаточный член о $(1)=\varepsilon_{n}(\boldsymbol{x})$ удовлетворяет соотношению

$$
\lim _{n \rightarrow \infty}\left|\varepsilon_{n}(\boldsymbol{x})\right|=0
$$

Нам понадобится следующее утверждение.

Лемма 3.3. Пусть выполнены условия теоремы 2.1A. Тогда для некоторых с $>0, C<\infty, n_{0}<\infty$ и для всех $n \geq n_{0}$ справедливо неравенство

$$
R(n):=\mathbb{P}\left(\mathbf{Z}(n)=\boldsymbol{x}, \tau_{\nu(n)+1} \geq \ln ^{2} n\right) \leq C n e^{-n D(\alpha)-c \ln ^{2} n} .
$$

Доказательство неравенства (3.13) в одномерном случае $d=1$ приведено в [11] (см. доказательство леммы 3.3 в [11]). Поскольку доказательство неравенства (3.13) в общем случае $d \geq 1$ повторяет (с очевидными изменениями) доказательство леммы 3.3 из [11], мы его опускаем.

Обозначим

$$
K_{m}(\boldsymbol{\alpha}):=e^{\lambda(\boldsymbol{\alpha}) m} \mathbb{P}(\tau \geq m)
$$

так что

$$
I_{Z}(\boldsymbol{\alpha})=\sum_{m \geq 1} K_{m}(\boldsymbol{\alpha})
$$

Лемма 3.4. В условиях теоремы $2.1 \mathrm{~A}$ найдется $\delta_{0}>0$ такое, что

$$
\sum_{m \geq 1} e^{\delta_{0} m} K_{m}\left(\boldsymbol{\alpha}_{0}\right)<\infty .
$$


Доказательство. Из экспоненциального неравенства Чебышева следует

$$
\mathbb{P}(\tau \geq m) \leq e^{-\Lambda_{\tau}(m)}
$$

где

$$
\Lambda_{\tau}(\theta):=\sup _{\lambda}\{\lambda \theta-A(\lambda, \mathbf{0})\}
$$

- функция уклонений для случайной величины $\tau$, которая удовлетворяет соотношению

$$
\liminf _{m \rightarrow \infty} \frac{1}{m} \Lambda_{\tau}(m) \geq \lambda_{+} .
$$

Поскольку в условиях теоремы $2.1 \mathrm{~A}$ выполняется $\lambda\left(\boldsymbol{\alpha}_{0}\right)<\lambda_{+}$, для некоторого $\delta_{0}>0$ и некоторого $C<\infty$ при всех $m \geq 0$ имеет место

$$
\mathbb{P}(\tau \geq m) \leq C e^{-2 \delta_{0} m} .
$$

Доказательство теоремь 2.1А. Имеем

$$
\begin{aligned}
P_{1}(n):= & \mathbb{P}\left(\mathbf{Z}(n)=\boldsymbol{x}, \tau_{\nu(n)+1} \leq \ln ^{2} n\right) \\
= & \mathbb{P}\left(\mathbf{Z}_{0}=\boldsymbol{x}, \nu(n)=0, \tau_{1} \leq \ln ^{2} n\right) \\
& +\sum_{k=1}^{\infty} \mathbb{P}\left(\mathbf{Z}_{k}=\boldsymbol{x}, \nu(n)=k, \tau_{\nu(n)+1} \leq \ln ^{2} n\right) \\
= & \mathbb{P}\left(\mathbf{Z}_{0}=\boldsymbol{x}, \tau_{1} \geq n, \tau_{1} \leq \ln ^{2} n\right) \\
& +\sum_{k=1}^{\infty} \mathbb{P}\left(\mathbf{Z}_{k}=\boldsymbol{x}, T_{k}<n \leq T_{k}+\tau_{k+1}, \tau_{k+1} \leq \ln ^{2} n\right) \\
= & 0+\sum_{k=1}^{\infty} \mathbb{P}\left(\mathbf{Z}_{k}=\boldsymbol{x}, T_{k}<n \leq T_{k}+\tau_{k+1}, \tau_{k+1} \leqslant \ln ^{2} n\right) \\
& \ln ^{2} n \sum_{m=1}^{\infty} \mathbb{P}\left(\mathbf{Z}_{k}=\boldsymbol{x}, T_{k}=n-m\right) \mathbb{P}\left(m \leq \tau \leq \ln ^{2} n\right) .
\end{aligned}
$$

Поскольку внутренняя сумма в правой части последней формулы «сворачивается» в функцию восстановления $H(n-m, \boldsymbol{x})$, мы получаем представление

$$
P_{1}(n)=\sum_{m=1}^{\ln ^{2} n} H(n-m, \boldsymbol{x}) \mathbb{P}\left(m \leq \tau \leq \ln ^{2} n\right) .
$$

Применяя далее теорему 2.3, находим из (3.16) представление

$$
P_{1}(n)=\psi_{1}\left(\lambda\left(\boldsymbol{\alpha}_{0}\right), \boldsymbol{\mu}\left(\boldsymbol{\alpha}_{0}\right)\right) \frac{C_{H}\left(1, \boldsymbol{\alpha}_{0}\right)}{n^{d / 2}} e^{-n D(1, \boldsymbol{\alpha})} P_{2}(n)(1+o(1)),
$$


где

$$
\begin{gathered}
P_{2}(n):=\sum_{m=1}^{\ln ^{2} n} K_{m}(\boldsymbol{\alpha}, n), \\
K_{m}(\boldsymbol{\alpha}, n):=\exp \{n(D(1, \boldsymbol{\alpha})-D(1-m / n, \boldsymbol{\alpha}))\} \mathbb{P}\left(m \leq \tau \leq \ln ^{2} n\right) .
\end{gathered}
$$

Очевидно, что для любого $m \in\left\{1,2, \ldots, \ln ^{2} n\right\}$ при $n \rightarrow \infty$ (см. (3.14)) имеет место сходимость

$$
K_{m}(\boldsymbol{\alpha}, n) \rightarrow K_{m}\left(\boldsymbol{\alpha}_{0}\right)
$$

и для любого $\delta>0$ и всех достаточно больших $n$ выполняются неравенства

$$
K_{m}(\boldsymbol{\alpha}, n) \leq e^{\delta m} K_{m}\left(\boldsymbol{\alpha}_{0}\right), \quad m \in\left\{1,2, \ldots, \ln ^{2} n\right\} .
$$

Поэтому, используя лемму 3.4 , в силу теоремы о мажорируемой сходимости получаем соотношение

$$
\lim _{n \rightarrow \infty} P_{2}(n)=\sum_{m=1}^{\infty} K_{m}\left(\boldsymbol{\alpha}_{0}\right)=I_{Z}\left(\boldsymbol{\alpha}_{0}\right) .
$$

Из соотношений (3.16)-(3.18) и леммы 3.3 вытекает утверждение теоремы 2.1A.

Доказательство теоремы 2.1*. Повторяя с очевидными изменениями вывод формулы (3.15), получаем

$$
\mathbb{P}(\mathbf{Z}(n)=\boldsymbol{x}, \gamma(n)=u)=H(n-u, \boldsymbol{x}) \mathbb{P}(\tau \geq u) .
$$

Применяя далее локальную теорему 2.3 для функции восстановления, получаем утверждение (2.12) теоремы 2.1*. Поскольку (2.13) доказывается аналогичным образом, теорема $2.1^{*}$ доказана.

Доказательство следствия 2.1. Докажем сначала, что выполнено равенство

$$
(n+1) D\left(\frac{\boldsymbol{x}}{n+1}\right)=n D(\boldsymbol{\alpha})+\lambda(\boldsymbol{\alpha})+o(1)
$$

Для

$$
\frac{\boldsymbol{x}}{n+1}=\frac{\boldsymbol{x}}{n}+\boldsymbol{y}=\boldsymbol{\alpha}+\boldsymbol{y}, \quad \text { где } \boldsymbol{y}:=\frac{-\boldsymbol{x}}{n(n+1)}
$$

имеем

$$
\begin{aligned}
(n+1) D\left(\frac{\boldsymbol{x}}{n+1}\right) & =n D\left(\frac{\boldsymbol{x}}{n}\right)+n\left(D\left(\frac{\boldsymbol{x}}{n+1}\right)-D\left(\frac{\boldsymbol{x}}{n}\right)\right)+D\left(\frac{\boldsymbol{x}}{n+1}\right) \\
& =n D(\boldsymbol{\alpha})+n(D(\boldsymbol{\alpha}+\boldsymbol{y})-D(\boldsymbol{\alpha}))+D(\boldsymbol{\alpha})+o(1) .
\end{aligned}
$$


В силу (2.5)

$$
D^{\prime}(\boldsymbol{\alpha})=\boldsymbol{\mu}(\boldsymbol{\alpha}), \quad \text { так что } n(D(\boldsymbol{\alpha}+\boldsymbol{y})-D(\boldsymbol{\alpha}))=-\boldsymbol{\mu}(\boldsymbol{\alpha}) \boldsymbol{\alpha}+o(1) .
$$

Поэтому, продолжая (3.20) и используя (2.5), получаем

$$
\begin{aligned}
(n+1) D\left(\frac{\boldsymbol{x}}{n+1}\right) & =n D(\boldsymbol{\alpha})-\boldsymbol{\mu}(\boldsymbol{\alpha}) \boldsymbol{\alpha}+\boldsymbol{\mu}(\boldsymbol{\alpha})+\lambda(\boldsymbol{\alpha})+o(1) \\
& =n D(\boldsymbol{\alpha})+\lambda(\boldsymbol{\alpha})+o(1) .
\end{aligned}
$$

Равенство (3.19) доказано. Для доказательства следствия 2.1 осталось воспользоваться теоремой 2.1, равенством (3.19) и соотношением

$$
\mathbf{Z}_{+}(n)=\mathbf{Z}(n+1) .
$$

3.4. Доказательство теорем 2.2, 2.2* и следствия 2.2. Приведем теперь эквивалентный вариант теоремы 2.2, который более удобен для доказательства.

Теорема 2.2A. Пусть выполнены условия $\left[\mathbf{C}_{0}\right]$ и $[\mathbf{Z}]$ и фиксирована точка

$$
\boldsymbol{\alpha}_{0} \in \mathfrak{A} \backslash \mathfrak{B}_{Y} .
$$

Пусть для начального случайного вектора $\boldsymbol{\xi}_{1}=\left(\tau_{1}, \boldsymbol{\zeta}_{1}\right)$ выполнено условие допустимой неоднородности

$$
\left(0, \boldsymbol{\mu}\left(\boldsymbol{\alpha}_{0}\right)\right) \in\left(\mathcal{A}_{1}\right), \quad\left(\lambda\left(\boldsymbol{\alpha}_{0}\right), \boldsymbol{\mu}\left(\boldsymbol{\alpha}_{0}\right)\right) \in\left(\mathcal{A}_{1}\right) .
$$

Тогда для любой последовательности $\boldsymbol{x}=\boldsymbol{x}_{n} \in \mathbb{Z}^{d}$ такой, что

$$
\lim _{n \rightarrow \infty} \boldsymbol{\alpha}=\boldsymbol{\alpha}_{0}, \quad \text { где } \boldsymbol{\alpha}:=\frac{\boldsymbol{x}}{n},
$$

имеет место представление (2.15), в котором остаточный член $о(1)=\varepsilon_{n}$ удовлетворяет соотношению

$$
\lim _{n \rightarrow \infty}\left|\varepsilon_{n}(\boldsymbol{x})\right|=0 .
$$

Нам понадобится следующее утверждение.

Лемма 3.5. Пусть выполнены условия теоремы $2.2 \mathrm{~A}$. Тогда найдутся константы $c>0, C<\infty, n_{0}<\infty$ такие, что для всех $n \geq n_{0}$ справедливо неравенство

$$
\mathbb{P}\left(\mathbf{Y}(n)=\boldsymbol{x},\left(\tau_{\eta(n)}, \boldsymbol{\zeta}_{\eta(n)}\right) \notin L_{n}^{d+1}, \eta(n) \geq 2\right) \leq C e^{-n D(\boldsymbol{\alpha})+c \ln ^{2} n},
$$

где

$$
L_{n}^{d+1}:=\left\{(m, \boldsymbol{z}) \in \mathbb{Z}^{d+1}: 1 \leq m \leq \ln ^{2} n, \max _{1 \leq i \leq d}\left|z_{i}\right| \leq \ln ^{2} n\right\} .
$$


Поскольку доказательство леммы 3.5 является почти дословным повторением доказательства леммы 2.3 в [14], мы его опускаем.

Лемма 3.6. Пусть выполнены условия теоремы 2.2А. Тогда найдется $\delta>0$ такое, что

$$
R\left(\boldsymbol{\alpha}_{0}, \delta\right):=\sum_{m \geq 1} e^{\left(\lambda\left(\boldsymbol{\alpha}_{0}\right)+\delta\right) m} \mathbb{E}\left(e^{\boldsymbol{\mu}\left(\boldsymbol{\alpha}_{0}\right) \boldsymbol{\zeta}+\delta|\boldsymbol{\zeta}|} ; \tau \geq m\right)<\infty .
$$

Доказательство. Если $\lambda\left(\boldsymbol{\alpha}_{0}\right) \geq 0$, то

$$
\begin{aligned}
R\left(\boldsymbol{\alpha}_{0}, \delta\right) & \leq \sum_{m \geq 1} e^{\delta m} \mathbb{E}\left(e^{\lambda\left(\boldsymbol{\alpha}_{0}\right) \tau+\boldsymbol{\mu}\left(\boldsymbol{\alpha}_{0}\right) \boldsymbol{\zeta}+\delta|\boldsymbol{\zeta}|} ; \tau \geq m\right) \\
& =\sum_{m \geq 1} e^{\delta m} \mathbb{E}\left(e^{\delta|\widehat{\boldsymbol{\zeta}}|} ; \widehat{\tau} \geq m\right)
\end{aligned}
$$

где распределение случайного вектора $(\widehat{\tau}, \widehat{\boldsymbol{\zeta}})$ определяется так:

$$
\mathbb{P}((\widehat{\tau}, \widehat{\boldsymbol{\zeta}}) \in \cdot):=\mathbb{E}\left(e^{\lambda\left(\boldsymbol{\alpha}_{0}\right) \tau+\boldsymbol{\mu}\left(\boldsymbol{\alpha}_{0}\right) \boldsymbol{\zeta}}:(\tau, \boldsymbol{\zeta}) \in \cdot\right) .
$$

В наших условиях выполняется $\left(\lambda\left(\boldsymbol{\alpha}_{0}\right), \boldsymbol{\mu}\left(\boldsymbol{\alpha}_{0}\right)\right) \in(\mathcal{A})$, поэтому для нового вектора $(\widehat{\tau}, \widehat{\boldsymbol{\zeta}})$ выполнено условие $\left[\mathbf{C}_{0}\right]$. Воспользуемся неравенством Коши: при достаточно малом $\delta>0$ и достаточно большом $C<\infty$ имеем

$$
\mathbb{E}\left(e^{\delta|\widehat{\boldsymbol{\zeta}}|} ; \widehat{\tau} \geq m\right) \leq\left(\mathbb{E} e^{2 \delta|\widehat{\boldsymbol{\zeta}}|}\right)^{1 / 2}(\mathbb{P}(\widehat{\tau} \geq m))^{1 / 2} \leq C(\mathbb{P}(\widehat{\tau} \geq m))^{1 / 2}
$$

Поскольку случайная величина $\widehat{\tau}$ удовлетворяет условию $\left[\mathbf{C}_{0}\right]$, найдутся константы $c>0$ и $C_{1}<\infty$ такие, что

$$
(\mathbb{P}(\widehat{\tau} \geq m))^{1 / 2} \leq C_{1} e^{-c m}
$$

для всех $m \geq 0$. Получили оценку

$$
R\left(\boldsymbol{\alpha}_{0}, \delta\right) \leq \sum_{m=1}^{\infty} C C_{1} e^{\delta m-c m}
$$

в которой правая часть конечна при $\delta \in(0, c)$.

Пусть теперь $\lambda\left(\boldsymbol{\alpha}_{0}\right)<0$. Тогда

$$
R\left(\boldsymbol{\alpha}_{0}, \delta\right) \leq \sum_{m \geq 1} e^{\delta m} \mathbb{E}\left(e^{\boldsymbol{\mu}\left(\boldsymbol{\alpha}_{0}\right) \boldsymbol{\zeta}+\delta|\boldsymbol{\zeta}|} ; \tau \geq m\right)
$$

и для оценивания правой части последнего неравенства можно воспользоваться неравенством Гёльдера: для любых $p, q \in(0,1)$ таких, что $p+q=1$, имеем

$$
R\left(\boldsymbol{\alpha}_{0}, \delta\right) \leq \sum_{m \geq 1} e^{\delta m}\left(\mathbb{E} e^{1 / p \boldsymbol{\mu}\left(\boldsymbol{\alpha}_{0}\right) \boldsymbol{\zeta}+1 / p \delta|\boldsymbol{\zeta}|}\right)^{p}(\mathbb{P}(\tau \geq m))^{q} .
$$


Поскольку в наших условиях выполняется $\boldsymbol{\mu}\left(\boldsymbol{\alpha}_{0}\right) \in(\mathcal{M})$, найдутся $\delta>0$, $c>0,1 / p>1$ и $C, C_{1}<\infty$ такие, что

$$
\left(\mathbb{E} e^{1 / p \boldsymbol{\mu}\left(\boldsymbol{\alpha}_{0}\right) \boldsymbol{\zeta}+1 / p \delta|\boldsymbol{\zeta}|}\right)^{p} \leq C, \quad(\mathbb{P}(\tau \geq m))^{q} \leq C_{1} e^{-c m}
$$

Поэтому из (3.24) выводим

$$
R\left(\boldsymbol{\alpha}_{0}, \delta\right) \leq \sum_{m \geq 1} e^{\delta m} C(\mathbb{P}(\tau \geq m))^{q} \leq \sum_{m \geq 1} e^{\delta m} C C_{1} e^{-c m}
$$

Поскольку правая часть последнего неравенства конечна при $\delta \in(0, c)$, неравенство (3.23) доказано.

Лемма 3.7. Пусть выполнены условия теоремы 2.2А. Тогда

$$
R_{1}(n):=\mathbb{P}(\mathbf{Y}(n)=\boldsymbol{x}, \eta(n)=1)=\mathbb{P}\left(\mathbf{Z}_{1}=\boldsymbol{x}, \tau \geq n\right)=o\left(\frac{1}{n^{d / 2}} e^{-n D(\boldsymbol{\alpha})}\right) .
$$

Доказательство утверждения леммы 3.7 повторяет доказательство части (b) леммы 4.1 в [14], поэтому мы его опускаем.

Доказательство теоремы 2.2A. Наряду с множеством $L_{n}^{d+1}$, определенным формулой (3.22), мы будем использовать множество

Имеем

$$
M_{n}^{d}:=\left\{\boldsymbol{z} \in \mathbb{Z}^{d} ; \max _{1 \leq i \leq d}\left|z_{i}\right| \leq \ln ^{2} n\right\}
$$

$$
\begin{aligned}
P(n) & :=\mathbb{P}\left(\mathbf{Y}(n)=\boldsymbol{x}, \boldsymbol{\xi}_{\eta(n)} \in L_{n}^{d+1}, \eta(n) \geq 2\right) \\
& =\sum_{k=2}^{\infty} \mathbb{P}\left(\mathbf{Z}_{k}=\boldsymbol{x}, \boldsymbol{\xi}_{k} \in L_{n}^{d+1}, \eta(n)=k\right) \\
& =\sum_{k=2}^{\infty} \mathbb{P}\left(\mathbf{Z}_{k}=\boldsymbol{x}, \boldsymbol{\xi}_{k} \in L_{n}^{d+1}, T_{k-1}<n \leq T_{k}\right) \\
& =\sum_{m=1}^{\ln ^{2} n} \sum_{\boldsymbol{z} \in M_{n}^{d}} \sum_{k=2}^{\infty} \mathbb{P}\left(\mathbf{Z}_{k-1}=\boldsymbol{x}-\boldsymbol{z}, T_{k-1}=n-m\right) \mathbb{P}(\tau \geq m, \boldsymbol{\zeta}=\boldsymbol{z}) .
\end{aligned}
$$

Поскольку внутренняя сумма в правой части последнего выражения «собирается» в функцию восстановления, получаем представление

$$
P(n)=\sum_{m=1}^{\ln ^{2} n} \sum_{\boldsymbol{z} \in M_{n}^{d}} H(n-m, \boldsymbol{x}-\boldsymbol{z}) \mathbb{P}(\tau \geq m, \boldsymbol{\zeta}=\boldsymbol{z}) .
$$


Применяя далее теорему 2.3, получаем

$$
P(n)=\psi_{1}\left(\lambda\left(\boldsymbol{\alpha}_{0}\right), \boldsymbol{\mu}\left(\boldsymbol{\alpha}_{0}\right)\right) \frac{C_{H}\left(1, \boldsymbol{\alpha}_{0}\right)}{n^{d / 2}} e^{-n D\left(1, \boldsymbol{\alpha}_{0}\right)} P_{1}(n, \boldsymbol{\alpha})(1+o(1)),
$$

где

$$
\begin{gathered}
P_{1}(n, \boldsymbol{\alpha}):=\sum_{m=1}^{\ln ^{2} n} \sum_{\boldsymbol{z} \in M_{n}^{d}} K_{m, \boldsymbol{z}}(n, \boldsymbol{\alpha}), \\
K_{m, \boldsymbol{z}}(n, \boldsymbol{\alpha}):=\exp \left\{n\left(D(1, \boldsymbol{\alpha})-D\left(1-\frac{m}{n}, \boldsymbol{\alpha}-\frac{\boldsymbol{z}}{n}\right)\right)\right\} \mathbb{P}(\tau \geq m, \boldsymbol{\zeta}=\boldsymbol{z}) .
\end{gathered}
$$

Легко видеть, что при $n \rightarrow \infty$

$$
K_{m, \boldsymbol{z}}(n, \boldsymbol{\alpha}) \rightarrow K_{m, \boldsymbol{z}}\left(\boldsymbol{\alpha}_{0}\right), \quad m \in\left\{1, \ldots, \ln ^{2} n\right\}, \quad \boldsymbol{z} \in M_{n}^{d},
$$

и для любого $\delta>0$ и всех достаточно больших $n$

$$
K_{m, \boldsymbol{z}}(n, \boldsymbol{\alpha}) \leq e^{\delta m+\delta|\boldsymbol{z}|} K_{m, \boldsymbol{z}}\left(\boldsymbol{\alpha}_{0}\right), \quad m \in\left\{1, \ldots, \ln ^{2} n\right\}, \quad \boldsymbol{z} \in M_{n}^{d} .
$$

Применяя далее лемму 3.6, в силу теоремы о мажорируемой сходимости получаем

$$
P_{1}(n, \boldsymbol{\alpha}) \rightarrow \sum_{m=1}^{\infty} \sum_{\boldsymbol{z} \in \mathbb{Z}^{d}} K_{m, \boldsymbol{z}}\left(\boldsymbol{\alpha}_{0}\right)=I_{Y}\left(\boldsymbol{\alpha}_{0}\right)
$$

Используя леммы 3.5, 3.7 и соотношения (3.25), (3.26), выводим

$$
\mathbb{P}(\mathbf{Y}(n)=\boldsymbol{x})=\psi_{1}\left(\lambda\left(\boldsymbol{\alpha}_{0}\right), \boldsymbol{\mu}\left(\boldsymbol{\alpha}_{0}\right)\right) \frac{C_{H}\left(1, \boldsymbol{\alpha}_{0}\right)}{n^{d / 2}} e^{-n D\left(\boldsymbol{\alpha}_{0}\right)} I_{Y}\left(\boldsymbol{\alpha}_{0}\right)(1+o(1)) .
$$

Утверждение (2.15) теоремы 2.2А и сама теорема 2.2A доказаны.

Доказательство теоремы 2.2* . Легко видеть, что для всех целых $v \geq 0$

$$
\mathbb{P}(\mathbf{Y}(n)=\boldsymbol{x}, \chi(n)=v)=\mathbb{P}(\mathbf{Z}(n+v+1)=\boldsymbol{x}, \gamma(n+v+1)=1) .
$$

Поэтому, вычисляя правую часть (3.27) с помощью теоремы $2.1^{*}$ и используя при этом соотношение

$$
-(n+v+1) D\left(\frac{\boldsymbol{x}}{n+v+1}\right)=-n D(\boldsymbol{\alpha})-(v+1) \lambda(\boldsymbol{\alpha})+o(1),
$$

которое вытекает из формулы (3.19), получаем соотношение (2.16).

Доказательство следствия 2.2 полностью аналогично доказательству следствия 2.1. 
3.5. Доказательство леммы 2.1. Установим равенства (2.18). Напомним, что для точек $\boldsymbol{\alpha}$ из некоторой окрестности точки $\boldsymbol{\alpha}_{0}=\boldsymbol{a}$ выполняется соотношение

$$
D(\boldsymbol{\alpha})=\boldsymbol{\mu}(\boldsymbol{\alpha}) \boldsymbol{\alpha}-A(\boldsymbol{\mu}(\boldsymbol{\alpha})),
$$

где функция $A(\boldsymbol{\mu})$ является решением уравнения

$$
\psi(-A(\boldsymbol{\mu}), \boldsymbol{\mu})=1
$$

а функция $\boldsymbol{\mu}(\boldsymbol{\alpha})$ является обратной к функции $A^{\prime}(\boldsymbol{\mu})$, т. е. удовлетворяет уравнению

$$
A^{\prime}(\boldsymbol{\mu}(\boldsymbol{\alpha}))=\boldsymbol{\alpha}
$$

Убедимся, что

$$
A(\mathbf{0})=0, \quad A^{\prime}(\mathbf{0})=\boldsymbol{a}, \quad A^{\prime \prime}(\mathbf{0})=B^{2} .
$$

Равенства (3.29) получаются из уравнения (3.28) с помощью дифференцирования его нужное число раз:

(0) при $\boldsymbol{\mu}=\mathbf{0}$ в силу $\psi(0, \mathbf{0})=1$ имеем $A(\mathbf{0})=0$;

(1) дифференцируя равенство (3.28) один раз при $\boldsymbol{\mu}=\mathbf{0}$, получаем

$$
\psi_{(1)}^{\prime}(0, \mathbf{0})\left(-A^{\prime}(\mathbf{0})\right)+\psi_{(2)}^{\prime}(0, \mathbf{0})=\mathbf{0},
$$

поэтому

$$
A^{\prime}(\mathbf{0})=\frac{\psi_{(2)}^{\prime}(0, \mathbf{0})}{\psi_{(1)}^{\prime}(0, \mathbf{0})}=\boldsymbol{a} ;
$$

(2) дифференцируя равенство (3.28) два раза при $\boldsymbol{\mu}=\mathbf{0}$, получаем

$$
\begin{aligned}
\psi_{(1,1)}^{\prime \prime} & (0, \mathbf{0})\left(-A^{\prime}(\mathbf{0})\right)\left(-A^{\prime}(\mathbf{0})\right)^{\top} \\
& -2 \psi_{(1,2)}^{\prime \prime}(0, \mathbf{0})\left(A^{\prime}(\mathbf{0})\right)^{\top}+\psi_{(2,2)}^{\prime \prime}(0, \mathbf{0})-\psi_{(1)}^{\prime}(0, \mathbf{0}) A^{\prime \prime}(\mathbf{0})=\mathbf{0 0}^{\top},
\end{aligned}
$$

поэтому

$$
A^{\prime \prime}(\mathbf{0})=\frac{1}{\psi_{(1)}^{\prime}(0, \mathbf{0})}\left[\mathbb{E} \zeta \zeta^{\top}-2 \boldsymbol{a} \mathbb{E} \boldsymbol{\zeta} \tau+\boldsymbol{a} \boldsymbol{a}^{\top} \mathbb{E} \tau^{2}\right]=B^{2} .
$$

Равенства (3.29) установлены. Поскольку функции $D^{\prime}(\boldsymbol{\alpha})$ и $A^{\prime}(\boldsymbol{\mu})$ являются взаимно обратными, из (3.29) следуют равенства

$$
D(\boldsymbol{a})=0, \quad D^{\prime}(\boldsymbol{a})=\mathbf{0}, \quad D^{\prime \prime}(\boldsymbol{a})=B^{-2} .
$$

Равенства (2.18) доказаны. 
Установим равенство (2.17). Поскольку

$$
I_{Z}(\boldsymbol{a})=\mathbb{E} \tau
$$

достаточно убедиться, что справедливо равенство

$$
C(\boldsymbol{a})=\frac{1}{\sigma \mathbb{E} \tau(2 \pi)^{d / 2}}, \quad \text { где } \sigma^{2}=\left|B^{2}\right|=\frac{1}{\left|D^{\prime \prime}(\boldsymbol{a})\right|} .
$$

Из закона больших чисел для процесса $\mathbf{Z}(n)$ и теоремы 2.1 следует, что найдется последовательность $\varepsilon_{n}>0$, стремящаяся к 0 достаточно медленно, для которой выполняется

$$
1=\lim _{n \rightarrow \infty} \mathbb{P}\left(\left|\frac{\mathbf{Z}(n)}{n}-\boldsymbol{a}\right|<\varepsilon_{n}\right)=C(\boldsymbol{a}) I_{Z}(\boldsymbol{a}) \lim _{n \rightarrow \infty} \Sigma_{n},
$$

где

$$
\Sigma_{n}:=\sum_{\boldsymbol{z} \in \mathbb{Z}^{d},|\boldsymbol{\alpha}-\boldsymbol{a}|<\varepsilon_{n}} \frac{1}{n^{d / 2}} e^{-n D(\boldsymbol{\alpha})} .
$$

Поскольку очевидно, что при $\varepsilon_{n} \sqrt{n} \rightarrow \infty$

$$
\begin{aligned}
\lim _{n \rightarrow \infty} \Sigma_{n} & =\lim _{n \rightarrow \infty} \int_{|\boldsymbol{u}|<\varepsilon_{n} \sqrt{n}} \exp \left\{-\frac{\boldsymbol{u} D^{\prime \prime}(\boldsymbol{a}) \boldsymbol{u}^{\top}}{2}\right\} d \boldsymbol{u} \\
& =\int_{\boldsymbol{u} \in \mathbb{R}^{d}} \exp \left\{-\frac{\boldsymbol{u} D^{\prime \prime}(\boldsymbol{a}) \boldsymbol{u}^{\top}}{2}\right\} d \boldsymbol{u}=\frac{(2 \pi)^{d / 2}}{\sqrt{\left|D^{\prime \prime}(\boldsymbol{a})\right|}}
\end{aligned}
$$

из (3.31) вытекает (3.30).

Авторы благодарят А. А. Боровкова за утверждение теоремы 2.1* и анонимного рецензента за очень доброжелательные и квалифицированные замечания.

\section{Список литературы}

1. Боровков A. A. Асимптотический анализ случайных блужданий. Быстро убывающие распределения приращений. М.: Физматлит, 2013.

2. Боровков А. А. Принципы больших уклонений в граничных задачах для обобщенных процессов восстановления // Сиб. матем. журн. 2016. T. 57, № 3, С. 562-595.

3. Боровков A. А., Могульский А. А. Вторая функция уклонений и асимптотические задачи восстановления и достижения границы для многомерных блужданий // Сиб. матем. журн. 1996. Т. 37, № 4 . C. $745-782$. 
4. Боровков А. А., Могульский А. А. Экспоненциальные неравенства чебышевского типа для сумм случайных векторов и для случайных блужданий // ТВП. 2011. Т. 56, № 1. С. 3-29.

5. Боровков A. А., Могульский А. А. Принципы больших уклонений для конечномерных распределений обобщенных процессов восстановления // Сиб. матем. журн. 2015. Т. 56, №1. С. 36-64.

6. Боровков A. А., Могульский А. А. Принципы больших уклонений для траектории обобщенных процессов восстановления. I // ТВП. 2015. T. 60, № 2. C. 227-247.

7. Боровков A. А., Могульский А. А. Принципы больших уклонений для траектории обобщенных процессов восстановления. II // ТВП. 2015. T. 60, № 3. C. 417-438.

8. Боровков А. А., Могульский А. А. Интегро-локальные предельные теоремы для обобщенных процессов восстановления при выполнении условия Крамера. I // Сиб. матем. журн. 2018. Т. 59, № 3. С. 491-513.

9. Боровков А. А., Могульский А. А. Интегро-локальные предельные теоремы для обобщенных процессов восстановления при выполнении условия Крамера. II // Сиб. матем. журн. 2018. Т. 59, № 4. С. 736-758.

10. Кокс Д. Р., Смит В. Л. Теория восстановления. М.: Изд-во «Советское радио», 1967.

11. Могульский A. А. Локальные теоремы для арифметических обобщенных процессов восстановления при выполнении условия Крамера // Сиб. электрон. матем. изв. 2019. Т. 16. С. 21-40.

12. Могульский А. А., Прокопенко Е. И. Интегро-локальные предельные теоремы для многомерных обобщенных процессов восстановления при моментном условия Крамера. I // Сиб. электрон. матем. изв. 2018. T. 15. C. $475-502$.

13. Могульский А. А., Прокопенко Е. И. Интегро-локальные предельные теоремы для многомерных обобщенных процессов восстановления при моментном условия Крамера. II // Сиб. электрон. матем. изв. 2018. T. 15. C. 503-527.

14. Могульский А. А., Прокопенко Е. И. Интегро-локальные предельные теоремы для многомерных обобщенных процессов восстановления при моментном условия Крамера. III // Сиб. электрон. матем. изв. 2018. T. 15 . C. $528-553$.

15. Могульский A. А., Прокопенко Е. И. Функция уклонений и базовая функция для многомерного обобщенного процесса восстановления // Сиб. электрон. матем. изв. 2019. Т. 16. С. 1449-1463. 
16. Asmussen S. and Albrecher H. Ruin Probabilities. 2nd ed. Hackensack, NJ: World Scientific, 2010.

Могульский Анатолий Альфредович

Прокопенко Евгений Игоревич

Институт математики,

им. С. Л. Соболева СО РАН,

просп. Академика Коптюга, 4,

Новосибирск, 630090 РОССИЯ.

Новосибирский гос. университет,

ул. Пирогова, 2,

Новосибирск, 630090 РОССИЯ.

E-mail: mogul@math.nsc.ru

E-mail: evgenii.prokopenko@gmail.com
Поступила в редакцию 4 февраля 2019 г.

Получена после доработки 8 мая 2019 г.

Принята к публикации 10 июня 2019 г. 